\title{
Empirical investigation of the signal performance of a high-resolution, indirect detection, active matrix flat-panel imager (AMFPI) for fluoroscopic and radiographic operation
}

\author{
L. E. Antonuk, Y. El-Mohri, J. H. Siewerdsen, J. Yorkston, \\ W. Huang, and V. E. Scarpine \\ Department of Radiation Oncology, University of Michigan Medical Center, Ann Arbor, Michigan 48109 \\ R. A. Street \\ Xerox Palo Alto Research Center, 3333 Coyote Road, Palo Alto, California 94304
}

(Received 17 June 1996; accepted for publication 30 October 1996)

\begin{abstract}
Signal properties of the first large-area, high resolution, active matrix, flat-panel imager are reported. The imager is based on an array of $1536 \times 1920$ pixels with a pixel-to-pixel pitch of $127 \mu \mathrm{m}$. Each pixel consists of a discrete amorphous silicon $n-i-p$ photodiode coupled to an amorphous silicon thin-film transistor. The imager detects incident $\mathrm{x}$ rays indirectly by means of an intensifying screen placed over the array. External acquisition electronics send control signals to the array and process analog imaging signals from the pixels. Considerations for operation of the imager in both fluoroscopic and radiographic modes are detailed and empirical signal performance data are presented with an emphasis on exploring similarities and differences between the two modes. Measurements which characterize the performance of the imager were performed as a function of operational parameters in the absence or presence of illumination from a light-emitting diode or $\mathrm{x}$ rays. These measurements include characterization of the drift and magnitude of the pixel dark signal, the size of the pixel switching transient, the temporal behavior of pixel sampling and the implied maximum frame rate, the dependence of relative pixel efficiency and pixel response on photodiode reverse bias voltage and operational mode, the degree of linearity of pixel response, and the trapping and release of charge from metastable states in the photodiodes. In addition, x-ray sensitivity as a function of energy for a variety of phosphor screens for both fluoroscopic and radiographic operation is reported. Example images of a line-pair pattern and an anthropomorphic phantom in each mode are presented along with a radiographic image of a human hand. General and specific improvements in imager design are described and anticipated developments are discussed. This represents the first systematic investigation of the operation and properties in both radiographic and fluoroscopic modes of an imager incorporating such an array. (C) 1997 American Association of Physicists in Medicine. [S0094-2405(97)01501-0]
\end{abstract}

Key words: digital x-ray imaging, active matrix flat-panel imager, amorphous silicon, indirect detection, signal measurements

\section{INTRODUCTION}

In recent years, a new class of real-time, digital x-ray imaging devices, based on the solid-state technologies developed for active matrix, flat-panel displays, has been undergoing development. These devices may be generically categorized as active matrix, flat-panel imagers (AMFPIs) since they all share the common characteristic of incorporating a twodimensional matrix of thin-film switches (one switch per pixel) of the same general type used in active matrix liquid crystal displays (AMLCDs).

In the majority of these imaging devices, the pixel switch is a single thin-film transistor (TFT) made from amorphous silicon $(a-\mathrm{Si}: \mathrm{H})^{1-6}$ although alternative approaches employing a cadmium selenide TFT, ${ }^{7}$ a single $a-\mathrm{Si}: \mathrm{H}$ diode, ${ }^{8-10}$ or dual $a$-Si:H diodes ${ }^{11}$ have also been reported. AMFPIs may further be divided into two categories depending upon whether the incident radiation is detected directly or indirectly. In the case of direct detection, the active matrix is coupled to a continuous, overlying layer of material which serves to convert the $\mathrm{x}$ rays into electron-hole pairs. ${ }^{12,13}$ This layer is of sufficient thickness to allow a significant fraction of the incident $\mathrm{x}$ rays to interact. Arrays thus far reported have used either $a-\mathrm{Si}: \mathrm{H}^{6}$ or cadmium selenide ${ }^{12}$ transistors coupled to an amorphous selenium $(a$-Se) converter. For the case of indirect detection, each pixel switch is coupled to either a discrete optical sensing element ${ }^{1}$ or, alternatively, to an overlying, continuous, optically sensitive layer. ${ }^{13,14}$ An $\mathrm{x}$-ray converter, such as a phosphor screen or CsI:Tl, is placed or deposited upon the array allowing the imaging pixels to sense light generated in the converter by interacting $\mathrm{x}$ rays. Thus far, optically sensitive arrays, which are compatible with the indirect detection approach, have primarily incorporated discrete $n-i-p$ or $p-i-n$ photodiodes coupled to either $a$-Si:H TFTs, ${ }^{1-4}$ to single diodes, ${ }^{8-10}$ or to back-toback diodes. ${ }^{11}$ There has also been an initial examination of $a$-Si:H TFTs coupled to discrete phototransistors ${ }^{5}$ and to a continuous photodiode layer. ${ }^{14}$ 
As in the case of AMLCDs, the pixel count of AMFPIs, led by the photodiode + TFT approach, has increased in an exponential manner since the creation of the first imaging arrays in $1988 .{ }^{15,16}$ Recently, our research on this approach has progressed to the point where a large-area, high spatial resolution array design has been realized. ${ }^{14,17}$ This array features a $1536 \times 1920$ pixel format with a $127 \mu \mathrm{m}$ pixel-topixel pitch. With an area of $19.5 \times 24.4 \mathrm{~cm}^{2}$, this device is the largest high resolution AMFPI thus far reported. ${ }^{17}$ This array is a slightly modified version of an earlier design in which pairs of gate lines were coupled at the peripheral contacts in order to facilitate easier electrical connection for initial evaluations. $^{15,18}$

This paper and a companion paper ${ }^{19}$ describe the results of an extensive investigation performed upon an imager incorporating the new $1536 \times 1920$ pixel high-resolution array along with acquisition electronics and various commercially available phosphor converters. While the performance of this prototype imager is far from optimal, the array and imager were created in the spirit of allowing an initial examination of the use of this technology for high resolution diagnostic imaging.

In this article, a detailed examination of the operation and signal performance of the imager is reported. Since the array itself is optically sensitive, the signal properties of the imager were found to be most efficiently examined through a combination of measurements in the absence of light ("dark" measurements), under illumination by a lightemitting diode light source ("LED" measurements), and under irradiation by a conventional x-ray source in the presence of an overlying phosphor screen (" $x$-ray" measurements). All results correspond to measurements from individual array pixels whose performance is representative of correctly functioning pixels on the array. [Spatial-frequencydependent signal investigations will be the subject of a future paper although preliminary $\operatorname{MTF}(f)$ results for a $127 \mu \mathrm{m}$ pixel array have previously been reported. $\left.{ }^{20}\right]$ The operational parameters (voltages, timings) required to run the imager are discussed in the context of various considerations, including array properties and external electronics, which govern the selection of these parameters. Measurements of a variety of pertinent signal properties are reported as a function of these operational parameters, irradiation conditions, and phosphor screen types. The properties investigated are those which define and/or limit the performance and practical use of the imager. In addition, example images of phantoms and of a human hand are presented and discussed in terms of imager properties and limitations. The implications of the measured signal properties are discussed and prospects for future improvements are summarized. The reported results represent the first such analysis for a large-area, high resolution TFT + photodiode AMFPI. In the companion paper, ${ }^{19}$ a theoretical and empirical investigation of the noise properties of such imagers, utilizing measured results reported in the present paper, is presented.

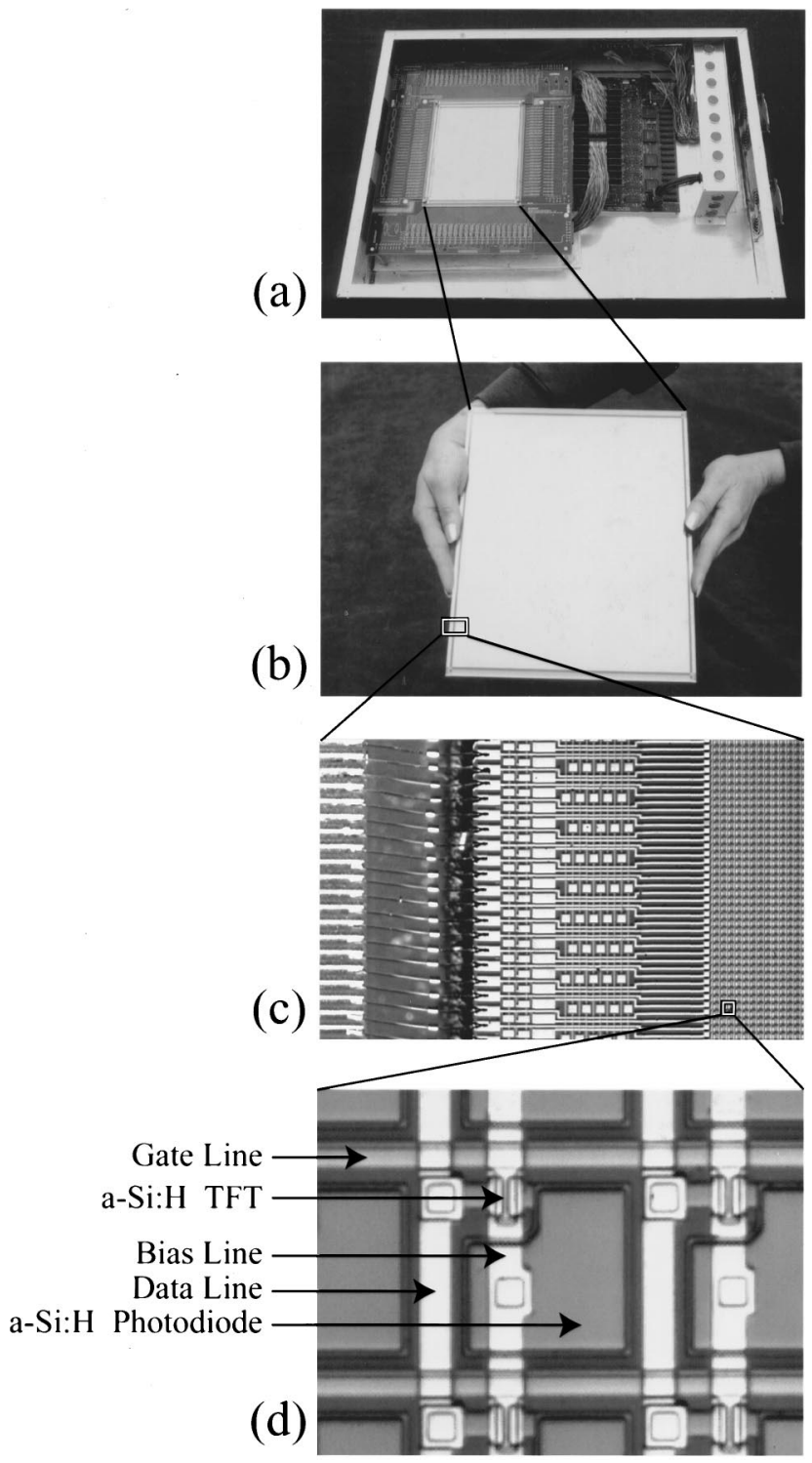

FIG. 1. Photographs of parts of the imaging system whose signal performance is reported in this article. (a) Photograph showing the array mounted to peripheral printed circuit boards which provide electrical contact to external acquisition electronics. (For the sake of clarity, the phosphor screen has been removed.) The gate driver and preamplifier circuits are located in close proximity to the array and all of these, along with the system logic board which directs the operation of the array, are housed in the aluminum box appearing in the picture. (b) Photograph of the array prior to wire bonding, illustrating its size compared to human anatomy. (c) Microphotograph of a portion of the array near the peripheral contacts. The wire bonds connecting the array to a printed circuit board are visible on the left-hand side. (d) Higher magnification microphotograph of a single pixel. Various features are labeled including a light-sensitive photodiode, a TFT, a bias rail which supplies a reverse bias voltage to the photodiodes, a gate line which is used to control the conductivity of TFTs and a data line which routes analog imaging signals from the pixels to external electronics.

\section{DESCRIPTION OF FLAT-PANEL IMAGER}

In this section, an overview of the structure and organization of the flat-panel imager reported in this paper is provided with an emphasis on novel features and enhancements. (Details of the construction of pixels and arrays of this general design and of the basic operation of earlier imagers have 


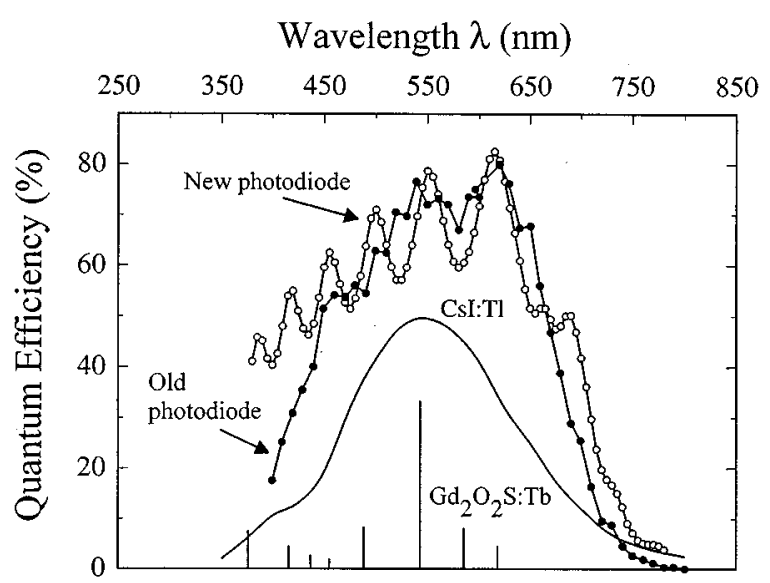

FIG. 2. Plot of the quantum efficiency of detection of light photons as a function of wavelength for n-i-p photodiodes. Data are presented for photodiodes of the type used in present arrays (open circles) as well as those of earlier, less optimized, arrays (Ref. 2) (solid circles). The oscillations in the data result from interference effects due to the passivation layer. The spectral output of $\mathrm{Gd}_{2} \mathrm{O}_{2} \mathrm{~S}: \mathrm{Tb}$ (Ref. 41) and CsI:Tl (Ref. 42), plotted in arbitrary units, is superimposed to illustrate the degree to which their output is utilized by the photodiodes.

been previously described in Refs. 2, 21, and 22.) In addition, the various operational parameters governing imager operation are summarized. Finally, in order to facilitate an understanding of both the significance of the imager proper- ties reported in this paper as well as the techniques used to examine them, a variety of considerations pertinent to the operation of the imager in fluoroscopic and radiographic modes are discussed.

\section{A. Structure and organization of imager}

The imaging system may be considered to consist of five major subsystems: (a) a $19.5 \times 24.4 \mathrm{~cm}^{2} a$-Si:H array with $1536 \times 1920$ pixels; (b) an overlying phosphor screen; (c) an external electronic acquisition system which provides a reverse bias voltage to $n-i-p$ photodiodes, controls signals to the array, and multiplexes, amplifies, and digitizes analog imaging signals from the pixels; (d) a host computer; and (e) the software which directs the general acquisition, analysis, and processing of the digitized array data.

Figure 1 illustrates the imager, the array, and magnified views of the array pixels. Compared to earlier large-area, 450 $\mu \mathrm{m}$ pitch arrays developed as prototypes for radiotherapy imaging $\left(256 \times 240\right.$ pixels giving a $11.5 \times 10.8 \mathrm{~cm}^{2}$ area $^{2}$ and $512 \times 560$ pixels giving a $23.0 \times 25.2 \mathrm{~cm}^{2}$ area ${ }^{23,24}$ ), a variety of significant improvements were incorporated into the design of the $127 \mu \mathrm{m}$ pitch array. As illustrated in Fig. 2, which shows the quantum efficiency of the photodiodes for light detection as a function of wavelength, photodiodes from the new arrays exhibit considerably enhanced performance at shorter wavelengths than for earlier arrays. Conse-

\begin{tabular}{|c|c|}
\hline \multicolumn{2}{|l|}{ Pixel } \\
\hline \multicolumn{2}{|l|}{ (n-i-p photodiode) } \\
\hline 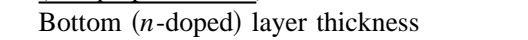 & $\sim 40-50 \mathrm{~nm} n^{+} a-\mathrm{Si}: \mathrm{H}$ \\
\hline Middle (intrinsic) layer thickness $\left(d_{\mathrm{pd}}\right)$ & $\sim 1.0-1.4 \mu \mathrm{m}$ intrinsic $a-\mathrm{Si}: \mathrm{H}$ \\
\hline Top ( $p$-doped) layer thickness & $\sim 10-20 \mathrm{~nm} p^{+}$microcrystalline-silicon \\
\hline Dimensions & $\sim 88 \mu \mathrm{m} \times 88 \mu \mathrm{m}$ [see Fig. $1(\mathrm{~d})]$ \\
\hline Photodiode light sensitive area & $\sim 5670 \mu \mathrm{m}^{2}$ \\
\hline \multicolumn{2}{|l|}{$(\underline{\mathrm{TFT}})$} \\
\hline 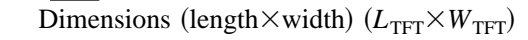 & $11 \mu \mathrm{m} \times 20 \mu \mathrm{m}$ \\
\hline TFT-on resistance $\left(R_{\text {TFT-on }}\right)$ & $\begin{array}{l}\sim 10 \times 10^{6} \Omega \text { at } V_{\text {TFT-on }}=+5 \mathrm{~V} \\
\sim 4 \times 10^{6} \Omega \text { at } V_{\text {TFT-on }}=+10 \mathrm{~V}\end{array}$ \\
\hline TFT leakage current & $\leqslant 1 \mathrm{fA}$ at $V_{\mathrm{TFT}-\text { off }}=-2$ to $-10 \mathrm{~V}$ \\
\hline \multicolumn{2}{|l|}{ Array } \\
\hline Pixel format (data $\times$ gate $)\left(N_{D} \times N_{G}\right)$ & $1536 \times 1920$ \\
\hline Pixel pitch & $127 \mu \mathrm{m}$ \\
\hline Array dimensions & $19.5 \times 24.4 \mathrm{~cm}^{2}$ \\
\hline Optical fill factor & 0.35 \\
\hline Data line capacitance & $\sim 50-100 \mathrm{pF}$ \\
\hline \multicolumn{2}{|l|}{ Electronics $^{\mathrm{a}}$} \\
\hline Preamplifiers & $\begin{array}{l}\text { Charge-sensitive circuit, } 1 \text { per data line, } 32: 1 \text { multiplexing } \\
\text { to ADC }\end{array}$ \\
\hline Gate drivers & Integrated serial display driver, 32 gate lines per chip \\
\hline Analog-to-digital converters & 18 bit digitization (trimmed to 15 bits) \\
\hline \multirow[t]{3}{*}{ System dark noise } & $\sim 4900 e$ (rms) uncorrelated \\
\hline & $\sim 4000 e(\mathrm{rms})$ correlated \\
\hline & $\sim 6300 e$ (rms) total system dark noise \\
\hline Packaging & Wire-bonding (array to surrounding printed-circuit cards) \\
\hline Controlling logic & Field-programable gate arrays + acquisition software \\
\hline
\end{tabular}

${ }^{\mathrm{a}}$ Reference 25 . 
TABLE II. Glossary of symbols relevant to operation of an array of the general design described in this paper. The table includes the values which are specific to the $127 \mu \mathrm{m}$ array examined as well as the range of operational parameters used to study the various properties.

\begin{tabular}{lll}
\hline \hline General & & \\
Dielectric constant for $a$-Si:H & $\kappa$ & $\sim 12$ \\
Permittivity of free space & $\epsilon_{0}$ & $8.85 \times 10^{-12} \mathrm{~F} / \mathrm{m}$ \\
Geometric area of photodiode & $A_{\mathrm{pd}}$ & $\sim 6780 \mu \mathrm{m}^{2}$ \\
Thickness of intrinsic layer of photodiode & $d_{\mathrm{pd}}$ & ${ }^{\mathrm{a}}$ \\
Width of TFT & $W_{\mathrm{TFT}}$ & $\mathrm{a}$ \\
Length of TFT & $L_{\mathrm{TFT}}$ & $\mathrm{a}$ \\
Field effect mobility for the TFT channel & $\mu_{\mathrm{fe}}$ & $0.5-1.0 \mathrm{~cm}^{2} / \mathrm{V} \mathrm{s}$ \\
Gate capacity per unit area for TFT & $\Gamma_{G}$ & $\sim 2 \times 10^{-8} \mathrm{~F} / \mathrm{cm}^{2}$ \\
Threshold voltage for TFT & $V_{T}$ & $\sim 0.5-2.5 \mathrm{~V}$ \\
Number of gate lines on an array & $N_{G}$ & \\
& & \\
Operational parameters & & \\
Photodiode reverse bias voltage & $V_{\text {bias }}$ & $0--6 \mathrm{~V}$ \\
TFT-on voltage applied to gate lines & $V_{\text {TFT-on }}$ & $2-10 \mathrm{~V}$ \\
TFT-off voltage applied to gate lines & $V_{\text {TFT-off }}$ & $-8 \mathrm{~V}$ \\
Duration TFT is kept conducting & $N_{\tau}$ & Units of $\tau_{\text {pix }}$ \\
Frame time & $T_{\text {frame }}$ & $15 \mathrm{~ms}$ to $\sim 30 \mathrm{~s}$ \\
Radiographic initialization time & $T_{\text {rad-init }}$ & $1-1000 \mathrm{~s}$ \\
\hline \hline
\end{tabular}

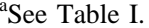

${ }^{b}$ While the characteristics of the array pixels require that the TFT be kept conducting for a period only $\sim 5-7$ times the length of the pixel time constant, $\tau_{\text {pix }}$ (i.e., only $20-35 \mu$ s for $V_{\text {TFT-on }}=5 \mathrm{~V}$ ), the design of the preamplifier circuit used for most of these measurements necessitated a considerably longer period which was fixed at $\sim 300 \mu \mathrm{s}$.

quently, increased light sensitivity is to be expected when the arrays are coupled to $\mathrm{x}$-ray converters having a significant output in the blue region of the spectrum. This new array incorporates finer minimum feature sizes permitted by more advanced processing tools and techniques. As a result, the linewidths were reduced from $\sim 25$ to $\sim 15 \mu \mathrm{m}$ and the gap between the gate (and data) lines and the photodiodes was reduced from $\sim 20$ to $\sim 8 \mu \mathrm{m}$. In addition, the TFT size was reduced from $240 \times 15 \mu \mathrm{m}^{2}$ (width $\times$ length) to $20 \times 11 \mu \mathrm{m}^{2}$ and repositioned from under the gate line to a corner within the region bounded by the gate and data lines. By virtue of these enhancements, an optical fill factor of 0.35 (the fraction of the surface of the array which is optically sensitive) was achieved for the new array. By comparison, the optical fill factor would have been less than 0.20 with the old design rules, even with a $20 \times 11 \mu \mathrm{m}^{2}$ TFT. In addition, a reduction in the overlap of the gate contact with the source (and drain) contact in the TFTs from $\sim 4$ to $\sim 3 \mu \mathrm{m}$, along with the reduced TFT width, resulted in a substantial diminution in the size of the pixel switching transient (defined and discussed below). To reduce gate line resistance on the array, a new bimetal material was used in place of chrome. This compensates for increased resistance due to the narrower lines thus helping to make the switching of TFTs almost temporally uniform across the array, as reported below. A further enhancement was the substitution of the top polyimide layer used on previous arrays with an oxynitride layer. This layer serves for planarization and passivation. The introduction of the oxynitride resulted in a large and crucial reduction in both the magnitude and temporal drift of the pixel dark signal, as reported below. Additional specifica- tions for the imager are listed in Tables I and II and a summary of symbols and equations which are relevant to the imager operation in the context of the current paper are given in Tables II and III.

The x-ray converters used for the measurements and images consisted of a collection of commercially available phosphor screens (Eastman Kodak, Rochester, NY). All screens consisted of a layer of gadolinium oxysulfide activated by terbium $\left(\mathrm{Gd}_{2} \mathrm{O}_{2} \mathrm{~S}: \mathrm{Tb}\right)$ mixed with a binder agent and deposited on an $\sim 0.18$-mm-thick plastic backing. The screens, along with their corresponding phosphor surface densities, were Lanex Fine $\left(\sim 34 \mathrm{mg} / \mathrm{cm}^{2}\right)$, Lanex Regular $\left(\sim 70 \mathrm{mg} / \mathrm{cm}^{2}\right)$, and Lanex Fast-B $\left(\sim 133 \mathrm{mg} / \mathrm{cm}^{2}\right)$. These screens were chosen due to the good overlap of the spectral sensitivity of the photodiodes with the spectral output of $\mathrm{Gd}_{2} \mathrm{O}_{2} \mathrm{~S}: \mathrm{Tb}$ (consisting of a number of discrete emission wavelengths, the most intense being at $545 \mathrm{~nm}$ ). This set of screens also provided a range of values for $\mathrm{x}$-ray quantum efficiency and light output.

Figure 1(a) shows a picture of the imaging array and the peripheral acquisition electronics. The electronic acquisition system is based on the same general architecture as a previously reported system ${ }^{25}$ although significant customization was performed to allow operation of the $127 \mu \mathrm{m}$ array. These modifications include expansion of the maximum number of addressable gate lines from 560 to 1920 and modification of the preamplification circuitry to allow sampling of the relatively small pixel signals from this array at a reduced system noise level. With these circuits, the total dark noise of the imaging system was $\sim 6300 e$ (rms). Of this total, $\sim 4900 e$ (rms) was due to uncorrelated noise while $\sim 4000 e$ (rms) was due to correlated noise believed to arise

TABLE III. Glossary of equations relevant to array operation. The equations assume SI units throughout. See Table II for definition of various symbols.

Sensor capacitance

$$
\begin{array}{cc}
C_{\mathrm{pd}}=\kappa \epsilon_{0} \frac{A_{\mathrm{pd}}}{d_{\mathrm{pd}}} & \text { (units of F) } \\
\text { Pixel charge capacity } & \\
Q_{\text {pix-cap }}=C_{\mathrm{pd}} V_{\text {bias }} & \text { (units of C) }
\end{array}
$$

TFT-on resistance

$$
R_{\mathrm{TFT}-\mathrm{on}}=\frac{1}{\left[\frac{W_{\mathrm{TFT}}}{L_{\mathrm{TFT}}} \mu_{\mathrm{fe}}\left(V_{\mathrm{TFT}-\text { on }}-V_{T}\right) \Gamma_{G}\right]} \quad \text { (units of } \Omega \text { ) }
$$

Sampling of pixel charge, $Q_{\text {pix }}$, by preamplifier circuit during readout

$$
Q_{\text {preamp }}(t)=Q_{\text {pix }}\left(1-e^{-\left(t / \tau_{\text {pix }}\right)}\right) \quad \text { (units of C) }
$$

Pixel time constant ${ }^{\mathrm{a}}$

$$
\tau_{\mathrm{pix}}=C_{\mathrm{pd}} R_{\mathrm{TFT}-\mathrm{on}} \quad \text { (units of } \mathrm{s} \text { ) }
$$

Minimum frame readout time and maximum theoretical frame rate ${ }^{\mathrm{b}}$

$$
\begin{array}{ll}
T_{\text {frame-min }}=N_{G} N_{\tau} \tau_{\text {pix }} & \text { (units of s) } \\
\mathrm{FPS}_{\text {max }}=\frac{1}{T_{\text {frame-min }}} & \text { (units of } \mathrm{s}^{-1} \text { ) }
\end{array}
$$

${ }^{\mathrm{a}}$ Reference 21

${ }^{\mathrm{b}}$ Reference 38 . 
from the power supplies for the photodiode bias voltage and for the gate drivers.

The present acquisition system allows a maximum of 512 consecutive data lines to be addressed (i.e., one-third of the array). Another limitation of the acquisition system is the relatively slow readout rate of $\sim 2.1 \mathrm{~ms}$ per gate line for 512 data lines. Consequently, the readout of a $512 \times 1920$ block of pixels requires $\sim 4 \mathrm{~s}$, although smaller pixel blocks can be read out considerably faster. Other parts of the system, not shown in Fig. 1(a), include the CAMAC crate and interface modules used to route signals between the system logic board and the host computer (a Macintosh 650 with 104 mbytes of random access memory). The basic synchronization of the control signals to the gate drivers and the preamplifier circuits is directed by logic embedded in fieldprogrammable gate arrays on the system logic board. Furthermore, a key feature of the system is that considerable control of the timing and addressing of the array is directed in real time by data acquisition software in the host computer. For example, relatively straightforward modifications of this software allowed synchronization of array timing with the LED and the $\mathrm{x}$-ray source as required.

\section{B. Operational parameters}

The array is controlled by a small number of operational parameters with typical ranges given in Table II. A reverse bias voltage, $V_{\text {bias }}$, is applied to the bias lines to create an electric field (and hence a depletion region) across the photodiodes and enhance signal collection efficiency. In addition, the pixel charge capacity, $Q_{\text {pix-cap }}$, is given by the product of $V_{\text {bias }}$ and the photodiode capacitance, $C_{\text {pd }}$, [Eqs. (1) and (2) in Table III]. During array operation, charge is allowed to accumulate in the capacitance of the photodiodes by keeping the TFTs in a nonconducting ("off"') state. This is accomplished by maintaining the gate lines at a negative voltage, $V_{\text {TFT-off }}$. For a given pixel, accumulation of charge decreases the electric field across the photodiode while increasing the potential difference between the source and drain contacts of the TFT. Readout (and simultaneous reinitialization) of a selected row of pixels is accomplished by applying a positive voltage, $V_{\mathrm{TFT}-\mathrm{on}}$, to the corresponding gate line. This renders the TFTs conducting ("on") with an on-resistance of $R_{\text {TFT-on }}$ [Eq. (3) in Table III]. The temporal behavior of the sampling of the pixel signal by the preamplifier is generally governed by a simple exponential function [Eq. (4) in Table III] with a time constant, $\tau_{\text {pix }}$, given by the product of $C_{\mathrm{pd}}$ and $R_{\text {TFT-on }}$ [Eq. (5) in Table III]. The degree to which the signal is sampled and the pixel is initialized is controlled by the time, $N_{\tau}$ (expressed in units of $\tau_{\text {pix }}$ ), that the TFTs are left conducting during the readout of each row of pixels.

The data acquired from a selected block of pixels on an array constitute a "frame" of information. For consecutive frames, the time between the readout of the same row of pixels is referred to as the frame time, $T_{\text {frame }}$. The minimum possible frame time, $T_{\text {frame-min }}$, is governed by the array properties and depends upon the product of the number of gate lines $\left(N_{G}\right), N_{\tau}$, and $\tau_{\text {pix }}$ [Eq. (6a) in Table III]. The maximum theoretical frame rate, FPS ${ }_{\max }$, is the inverse of $T_{\text {frame-min }}$ [Eq. (6b) in Table III]. The final operational parameter appearing in Table II, $T_{\text {rad-init }}$, is discussed in Sec. III.

\section{Considerations for fluoroscopic and radiographic imager operation}

For the purposes of this paper, we define two modes of image acquisition. "Fluoroscopic mode" will refer to the acquisition of a series of consecutive image frames during either a continuous wave (cw) irradiation or a pulsed irradiation. (In this paper, an "image frame" shall be defined to correspond to data acquired when the array is exposed to LED light or x-ray radiation as opposed to data acquired in the absence of any form of illumination.) "Radiographic mode" will refer to the acquisition of a single image frame following a brief irradiation. (For purposes of examining the imager properties, the "radiation" source can, in some cases, be either an x-ray source or a light source, e.g., a LED.) A variety of considerations, which can be different for radiographic and fluoroscopic mode, govern the selection of the values for the operational parameters used to control the array. In this section, a number of these considerations are briefly outlined, and in the following sections measurements are reported which illustrate and quantify their effect upon the signal performance of the imager.

\section{Photodiode and TFT leakage current}

The reverse bias voltage applied to the photodiodes results in a dark (leakage) current. ${ }^{26}$ In addition, the finite (though very high) resistance of the TFT in the nonconducting state allows an opposing leakage current. ${ }^{27}$ Since photodiode leakage increases with the voltage across the photodiode, it decreases as the pixel acquires signal during a frame. Conversely, TFT leakage increases slowly with increasing pixel signal due to the increasing potential difference across the transistor. The net result of these two effects is referred to as the pixel dark current. The magnitude and long-term temporal variation in the dark current have an important influence upon the behavior of the imager and it is generally desirable that both be as small as possible. For example, when the TFTs are in the nonconducting state, under some operating conditions the photodiode dark current dominates and there is a steady accumulation of dark signal in the pixel which decreases the electric field across the photodiode. If the pixel is not read out, this process continues until the two leakage currents reach equilibrium. Thus, the accumulation of dark signal can result in partial or complete loss of useful signal capacity in the pixel and, for sufficient reduction of field strength, reduced signal collection efficiency. Under other conditions, there can be a steady loss of signal from the pixel due to the net pixel dark current. Both cases are observed and their effects upon fluoroscopic and radiographic performance are illustrated and quantified below. 
(a)

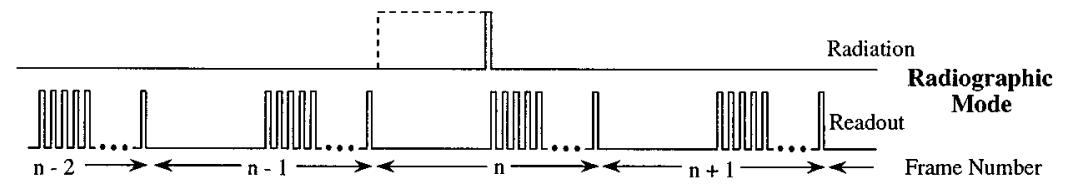

(b)

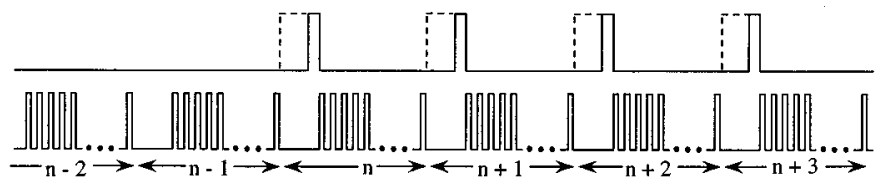

\section{Pulsed}

Fluoroscopic

Mode

(c)
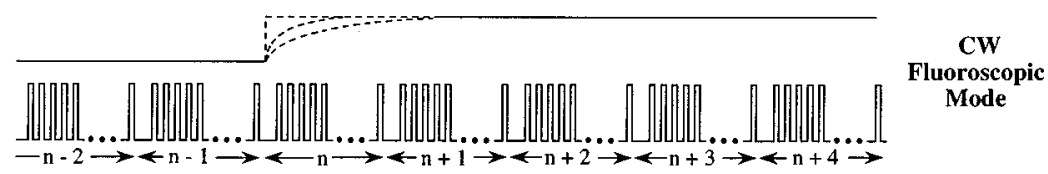

FIG. 3. Schematic illustration of the synchronization of timing between the flat-panel imager and the radiation source for a variety of cases. In each case, the relative timing of the delivery of the radiation (top) and the readout of the $N_{G}$ individual gate lines for the corresponding frame(s) (bottom) is shown. The frames are numbered with respect to the first image frame (" $n$ "). (a) Radiographic mode illustrating both a short (solid line) and long (dashed line) radiation pulse. (b) Fluoroscopic mode with the radiation pulsed once per frame. (c) Fluoroscopic mode with continuous wave radiation where various degrees of "ramping" of the radiation output is illustrated by the dashed lines.

\section{Pixel switching transients}

Another consideration concerns the effects of switching the gate line voltage to turn the TFTs on and off. This switching action generates pixel switching transients as a result of capacitive coupling including that between the gate and data lines, between the gate lines (and the data lines) and the photodiodes, and between the gate and source (and gate and drain) contacts in the TFTs. When the gate line voltage is made positive in order to turn the TFTs on, a positive transient is presented to the preamplifier circuit (whose sampling typically starts slightly before the switching so as not to lose any imaging signal). As this transient is opposite in polarity to the pixel imaging signal, it is necessary that the preamplifier circuit be designed to compensate for it in order to avoid loss of information. When the gate line voltage is made negative to turn the TFTs off, a negative transient is presented to the photodiode. This results in an effective reduction in the reverse bias voltage (and hence, the electric field) across the photodiode. This effect may be compensated for by increasing the magnitude of $V_{\text {bias }}$. (The preamplifier senses a negative transient only if its sampling is not terminated before this voltage change.) The size of these effects should be quantified to properly construct the preamplifier circuit and to knowledgeably adjust $V_{\text {bias }}$. Moreover, it is generally desirable to minimize the size of the pixel switching transients to lessen the constraints upon preamplifier design. This is achieved through reduction of capacitance in the array and by minimizing the difference between $V_{\text {TFT-on }}$ and $V_{\text {TFT-off }}$.

\section{Synchronization with radiation source}

For a variety of reasons, synchronization of the imager and the radiation source is often an important consideration. These include the following: to assure complete use of the radiation signal; to minimize dark current effects; and to en- sure consistency between the timing conditions used to acquire image processing constants and those used for images. Figure 3 schematically illustrates such synchronization for a variety of cases. In the case of radiographic mode, a single brief irradiation is precisely timed to occur between frames. Figure 3(a) illustrates this case where consecutive gate lines are read out as quickly as possible following a fixed-length pause at the beginning of each frame to accommodate the full range of irradiation periods provided by the source (solid and dashed lines). In the case of a pulsed radiation source for fluoroscopy, the delivery of each pulse is also synchronized with the interval between frames. In addition, for fluoroscopic units which vary the radiation amount by varying the pulse width, a fixed-length pause may be provided at the beginning of each frame to accommodate the full range of pulse widths, as illustrated in Fig. 3(b). Figure 3(c) illustrates a final case where fluoroscopic mode is performed with a $\mathrm{cw}$ source. In this case, the start of the radiation is precisely timed to occur between frames as in the previous two cases. The degree to which the synchronization shown in Fig. 3(c) is useful depends, at least, upon how quickly the source increases to full intensity. In the event that this "ramp-up" time is significant compared to the frame time [see the dashed lines in Fig. 3(c)], the first image frames will not have a constant radiation signal presented to all parts of the array. In this case, there is little motivation for providing exact (or any) synchronization between the imager and the radiation source.

\section{The effects of trapping states}

The existence of deep metastable ("trapping") states between the conduction and valence energy bands in $a-\mathrm{Si}: \mathrm{H}$, and their high density (a minimum of $\sim 3 \times 10^{15} / \mathrm{cm}^{3}$ for high quality $a-\mathrm{Si}: \mathrm{H}$ ), have a significant effect upon the properties of $a$-Si:H photodiodes and TFTs ${ }^{26,28,29}$ and, hence, on imag- 
ers incorporating these devices. In the case of the TFTs, these states are responsible for very low mobilities for charge transport resulting in rather large on and off resistances, $\sim 10^{6} \Omega$ [from Eq. (3)] and $\sim 10^{15} \Omega,{ }^{29}$ respectively. In addition, when a TFT is initially switched off, it generates a slowly decaying current due to the release of trapped charge- essentially a transient TFT current. ${ }^{29}$ In the case of the photodiodes, the trapping and release of charge by the trapping states is manifest in a variety of ways. For example, when $V_{\text {bias }}$ is first applied, or when the pixels are first read out after having been allowed to saturate, the (re)establishment of the electric field across the photodiode results in the photodiode dark current unavoidably exhibiting a measurable initial (decaying) transient behavior. This transient may last from seconds to several minutes or longer, depending upon the quality and the construction of the photodiode. Another effect is the capture of some fraction of the imaging signal (electrons) in trapping states in the photodiode when an array is irradiated. The amount of trapped signal increases as the electric field strength across the photodiode decreases. ${ }^{30}$ This occurs if $V_{\text {bias }}$ is decreased and/or if the incident light signal, and hence the pixel signal size, increases. In radiographic mode, this trapping has the negative consequence of essentially reducing the useful optical imaging quanta. In fluoroscopic mode, during the initial image frames the charge captured by the traps is gradually balanced by the release of trapped charge until an approximate equilibrium is achieved. As a result, these initial frames exhibit a strong visual artifact as the images progressively show more signal. This capture and release of charge also results in the carryover of charge between fluoroscopic image frames resulting in image lag. However, a small amount of carryover is tolerable and, in fact, can improve the quality of the imaging due to reduction of noise. ${ }^{19,31}$

\section{Linearity of pixel response}

The response of the pixels to incident radiation is initially linear at small signal levels. As signal size grows, the electric field across the photodiode decreases which increases the effects of charge trapping and eventually saturation, thus leading to a progressive loss of linearity. The fraction of the pixel signal range over which the response of the pixel remains highly linear increases with $V_{\text {bias }}$, since this reduces the amount of charge trapping and extends the point at which saturation effects are manifest. ${ }^{32,33}$ Thus, extending the pixel signal capacity by increasing $V_{\text {bias }}$ also has the desirable consequence of increasing the range of linear response. For example, in a region of linear response, image processing constants which correct for pixel-to-pixel variations in dark signal and light response and for channel-to-channel variations in preamplifier offset and gain may be based upon a simple two-parameter fit of dark and flood field measurements. ${ }^{2}$

\section{Selection of operating parameters}

The selection of the values for $V_{\text {bias }}, V_{\text {TFT-on }}, V_{\text {TFT-off }}$, and $T_{\text {frame }}$ are subject to a variety of requirements, some of which conflict. The size of the imaging signal, which depends upon the $\mathrm{x}$-ray energy, exposure to the detector, and screen type, is a key determinant of the required pixel signal capacity, and hence of $V_{\text {bias }}$. While, it is desirable to keep $V_{\text {bias }}$ large for reasons of high light detection efficiency, large pixel signal capacity, reduced charge trapping, and improved linearity, this is opposed by the desire to keep $V_{\text {bias }}$ low to minimize the magnitude and temporal drift of the photodiode dark current. It is generally desirable to keep $V_{\text {TFT-on }}$ large in order to reduce the frame time [Eqs. (3), (5), (6a)] while it is also desirable to reduce the difference between $V_{\text {TFT-on }}$ and $V_{\text {TFT-off }}$ to minimize the pixel switching transients. Finally, the magnitude of $V_{\text {TFT-off }}$ must be kept larger than that of $V_{\text {bias }}$, typically by $\sim 2-4 \mathrm{~V}$. In the event that the signal size brings the pixel near saturation, this strategy prevents the leakage of charge from the photodiode through the TFT onto the data line. Such charge leakage would result in a loss of image contrast. For a given application, the selection of a set of operational parameters which represents a satisfactory compromise between these competing considerations must be based upon a knowledge of the magnitude and systematics of the relevant imager properties. In the following sections, measurements which are pertinent to the choice of the operational parameters and which highlight the similarities and differences between operating the imager in fluoroscopic and radiographic modes are reported.

\section{MOTIVATIONS AND METHODS}

In the first part of this section, experimental methods common to the various studies are summarized. In the latter part, further details specific to each of the studies, along with specific motivations for these studies, are concisely described in a series of subsections.

Array measurements were performed in the dark, with light from a LED, and with $\mathrm{x}$ rays. The measurements with light were performed by directly illuminating the array with a flashing LED source. This LED has a central emission wavelength of $\sim 568 \mathrm{~nm}$, near the peak of the photodiode efficiency. Under control by the acquisition software, the LED was turned on ("flashed") for $\sim 2.8 \mu$ s every $\sim 5.6 \mu \mathrm{s}$. Gross control of the amount of light shining on the array pixels was achieved through careful positioning of the LED above the array and systematic variation of the amount of light was achieved by flashing the LED a specified number of times between frames. X-ray measurements were performed using a Simulix Y therapy simulator (Oldelft, Fairfax, VA) with a Maxiray 100 tube source (GE Medical Systems, Milwaukee, WI). This unit provided both radiographic $\mathrm{x}$-ray pulses and $\mathrm{cw}$ fluoroscopic radiation. For fluoroscopic operation, the ramp-up and the "ramp-down" times (the time to completely turn on and off) were $\sim 30$ and $\sim 60 \mathrm{~ms}$, respectively. Synchronization of the operation of the imager with the delivery of radiation for radiographic mode measurements was achieved by the addition of a logic circuit to the control system of the simulator. This circuit made it possible for the imager acquisition software to trigger individual radiographic $\mathrm{x}$-ray pulses. 
TABLE IV. Summary of the experimental conditions or parameters used for the measurements. These include whether the radiation source was a LED, $x$-ray tube, or if the measurements were performed in the dark. For measurements with the LED or $\mathrm{x}$ rays, the mode of operation is specified (fluoroscopic or radiographic). The column labeled "Sync" refers to whether the readout of the array was synchronized with the radiation source. The range of values for the operational parameters $\left(V_{\text {bias }}, V_{\text {TFT-on }}, T_{\text {frame }}\right.$, and $\left.T_{\text {rad-init }}\right)$ is also presented. $V_{\text {TFT-off }}$ and $\left(N_{\tau} \tau_{\text {pix }}\right)$ were fixed at the values given in Table II. Note that the column labeled $T_{\text {rad-init }}$ applies only for radiographic mode and for this case $T_{\text {frame }}$ was fixed at $33 \mathrm{~ms}$.

\begin{tabular}{|c|c|c|c|c|c|c|c|c|}
\hline Property & Source & Mode & Sync & $\begin{array}{l}V_{\text {bias }} \\
(\mathrm{V})\end{array}$ & $\begin{array}{l}V_{\text {TFT-on }} \\
(\mathrm{V})\end{array}$ & $\begin{array}{l}T_{\text {FRAME }} \\
(\mathrm{s})\end{array}$ & $\begin{array}{l}T_{\text {RAD-INIT }} \\
(\mathrm{s})\end{array}$ & $\begin{array}{l}\text { Figure } \\
\text { /Table }\end{array}$ \\
\hline (A) Pixel dark signal drift & Dark & $\cdots$ & $\cdots$ & $-2,-4,-6$ & 5 & 1.4 & $\cdots$ & Fig. 4 \\
\hline (B) Pixel dark signal & Dark & $\cdots$ & $\cdots$ & $-2,-6$ & 5 & 0.015 to 30 & $\cdots$ & Fig. 5 \\
\hline (C) Pixel switching transient & Dark & $\ldots$ & $\ldots$ & -4 & 2 to 10 & 1.4 & $\cdots$ & Fig. 6 \\
\hline $\begin{array}{l}\text { (D) Temporal behavior of pixel } \\
\text { sampling }\end{array}$ & LED & Fluor & Yes & -4 & 2 to 10 & 0.015 & $\cdots$ & Fig. 7 \\
\hline (E) Relative pixel efficiency & LED & Fluor/Rad & Yes & 0 to -6 & 5,10 & 0.033 to 1 & 0.5 to 10 & Fig. 8 \\
\hline (F) Pixel response and linearity & LED & Fluor/Rad & Yes & -2 to -6 & 5 & 0.033 to 1 & 0.34 to 20 & Figs. 9,10 \\
\hline (G) Pixel charge trapping & LED & $\ldots$ & Yes & $-2,-4,-6$ & 5 & 0.033 & 1 to 1000 & Fig. 11 \\
\hline Pixel charge release & LED & $\ldots$ & Yes & $-2,-4,-6$ & 5 & 0.020 to 2 & $\ldots$ & Fig. 12 \\
\hline (H) Pixel x-ray sensitivity & $\mathrm{X}$ ray & Fluor/Rad & $\mathrm{No} / \mathrm{Yes}$ & -4 & 5 & 0.2 & 5 & Table V \\
\hline (I) X-ray images & $\mathrm{X}$ ray & Fluor/Rad & $\mathrm{No} / \mathrm{Yes}$ & -4 & 5 & $\sim 4$ & 80 & Figs. 13,14 \\
\hline
\end{tabular}

A summary of experimental conditions and operational parameters varied during the measurements is presented in Table IV. For the studies of dark signal drift, dark signal, and pixel switching transient, the measurements were performed in the dark. For the dark signal studies, the imager is considered to be operating neither in the fluoroscopic nor radiographic mode; the array is simply read out continually in the absence of illumination. The studies of charge trapping and release involved methodologies consisting of measurements utilizing both fluoroscopic and radiographic modes and in the dark. For the studies of temporal behavior of pixel sampling, relative efficiency, pixel response, linearity of response, and charge trapping and release, the LED was used as it provided a highly stable source, offered precise and brief turn-on and turn-off times, and allowed accurate variation of the amount of signal. For most of the measurements requiring light or $\mathrm{x}$ rays, results are reported for both fluoroscopic and radiographic modes. However, since the pixel temporal sampling behavior does not depend on mode, results are reported only for fluoroscopic mode.

For the studies in which the imager was operated in fluoroscopic mode (Table IV), the measurements were performed under conditions in which equilibrium had been established between the trapping and release of charge. This was accomplished by excluding data from initial image frames during which the signal, for reasons explained in the previous section, was increasing. Thus, for a given set of operational parameters $\left(V_{\text {bias }}, V_{\text {TFT-on }}, V_{\text {TFT-off }}, N_{\tau}, T_{\text {frame }}\right)$, a typical data acquisition sequence consisted of: (a) reading the array in the dark for up to 50 frames (for purposes of initialization), followed by (b) acquiring a consecutive set of $\sim 70$ image frames with data from the last $\sim 20$ image frames being analyzed and averaged. By excluding data taken under nonequilibrium conditions where the effects of charge trapping and release were varying, this strategy contributed toward the precision and reproducibility of the results.

For the studies in which the imager was operated in radiographic mode (Table IV), the effects of charge trapping also necessitated the use of a particular methodology to en- sure precise, reproducible results. The method involved taking a consecutive series of radiographic image frames each of which was preceded by a period during which the array was continually read out in the dark. The readout period between each pair of radiographic image frames is defined as the radiographic initialization time, $T_{\text {rad-init }}$. Thus, for a given set of operational parameters $\left(V_{\text {bias }}, V_{\text {TFT-on }}, V_{\text {TFT-off }}, N_{\tau}\right.$, $\left.T_{\text {frame }}, T_{\text {rad-init }}\right)$, a typical data acquisition sequence consisted of the following sequence repeated ten times without pause: (a) reading the array in the dark for a time $T_{\text {rad-init }}$, followed by (b) reading a radiographic image frame. (In some instances, reproducibility of the results was found to be further improved by preceding this sequence by reading the array out in the dark for an additional, limited duration.) As the signal size was observed to increase asymptotically by up to $5 \%$ over the first few image frames (establishing a form of equilibrium), only the results from the last five image frames were analyzed and averaged. This methodology (and that described above for fluoroscopic mode studies) resulted in a reproducibility of better than $1 \%$ throughout the measurements. In practical terms, properties reported for a given value of radiographic initialization time using the above methodology approximately correspond to the behavior to be expected from an imager operated under conditions where patient images are made, on average, every $T_{\text {rad-init }}$ seconds (where the duration of $T_{\text {rad-init }}$ could range from a few seconds to tens of minutes or longer depending on the application). Moreover, as reported below, for a given value of radiographic initialization time, the properties measured in radiographic mode were found to be independent of frame time, thus making $T_{\text {rad-init }}$ (and not $T_{\text {frame }}$ ) the more fundamental independent variable for radiographic mode.

For many of the reported studies, measurements were performed as a function of one or more of the operational parameters, $V_{\text {bias }}, V_{\text {TFT-on }}, T_{\text {frame }}$, and $T_{\text {rad-init }}$ over the ranges indicated in Table IV. $V_{\text {bias }}$ and $V_{\text {TFT-on }}$ were otherwise fixed at values of -4 and $5 V$, respectively and the TFTs were kept conducting for $\sim 300 \mu$ s during readout (see Table II). For the radiographic measurements, unless otherwise indi- 
cated, $T_{\text {frame }}$ was fixed at $33 \mathrm{~ms}$ for all values of $T_{\text {rad-init }}$. $V_{\text {TFT-off }}$ was fixed at $-8 \mathrm{~V}(2 \mathrm{~V}$ below the most negative value used for $V_{\text {bias }}$ ). Given the relatively slow acquisition rate of the electronics, data were read out from small, contiguous pixel blocks, typically $64 \times 20$ (data $\times$ gate lines) to allow frame times, $T_{\text {frame }}$, as short as $\sim 15 \mathrm{~ms}$ corresponding to a frame rate of $\sim 60 \mathrm{fps}$. Although nonaddressed pixels on the array would saturate during the measurements, this had no effect (i.e., cross talk) on measurements from the addressed pixels. This was empirically confirmed by performing a limited number of measurements in which both $64 \times 20$ pixels and $64 \times 1920$ pixels were addressed under otherwise identical operational conditions. The very high degree of isolation between pixels on different data lines prevents cross talk between these lines. In addition, the aforementioned strategy of maintaining the magnitude of $V_{\text {TFT-off }}$ higher than $V_{\text {bias }}$ ensures negligible cross talk between pixels along the same data line. All measurements were carried out at a constant temperature of $\sim 28{ }^{\circ} \mathrm{C}$, which was $\sim 4{ }^{\circ} \mathrm{C}$ above room temperature due to heat generated by the acquisition electronics which are located in close proximity to the array. In addition, the correspondence between a change in charge presented to the input of the preamplifiers and a change in digitizer output [analog-to-digitizer (ADC) value] (i.e., pC per ADC unit) was determined through a separate calibration of the acquisition electronics.

For all measurements involving the LED source (both fluoroscopic and radiographic) and for radiographic x-ray measurements and imaging, the operation of the imager was synchronized with the radiation source in the general manner illustrated in Figs. 3(a) and 3(b). For fluoroscopic x-ray operation, no synchronization was used as there was no advantage to be gained. For all measurements, the time to read out each row of 64 pixels was fixed $(\sim 0.7 \mathrm{~ms}$ per row). In order to vary $T_{\text {frame }}$, a pause was introduced between the readout of the last gate line for a given frame and the first gate line for the next frame. The LED flashes or radiographic $\mathrm{x}$-ray pulse occurred at the end of this pause as illustrated in Figs. 3(a) and 3(b).

\section{A. Pixel dark signal drift}

An important indicator of the quality of the pixels is the degree to which the pixel dark signal, $Q_{\text {pix-dk }}$, remains stable over extended periods of time. (The stable operation of the acquisition electronics over extended periods was independently verified.) For each selected value of $V_{\text {bias }}$ and with all other operational parameters fixed, the dark signal drift was examined by reading out the array continuously for up to $8 \mathrm{~h}$ after $V_{\text {bias }}$ was applied. Over this time, data from selected pixels were periodically saved. Due to the fact that the preamplifier circuit contributes an unknown constant offset to the total pixel signal, the empirical results do not provide an absolute measure of the dark signal but, nevertheless, allow an examination of its variation over time.

\section{B. Pixel dark signal}

The variation of the magnitude of the pixel dark signal was examined as a function of $T_{\text {frame }}$ and $V_{\text {bias }}$. From these data, a measure of the absolute magnitude of the pixel dark current, equal to the slope of the dark signal versus $T_{\text {frame }}$ behavior, was obtained.

\section{Pixel switching transient}

As mentioned above, switching the gate line voltage between $V_{\text {TFT-on }}$ and $V_{\text {TFT-off }}$ during array operation induces switching transients which are presented to the preamplifier circuits and the photodiodes. A quantification of the magnitude of these transients is useful as they affect the size of the electric field across the photodiode as well as the design of the preamplifier. In our acquisition system, during normal operation (method A) charge integration on the feedback capacitance of the preamplifiers begins shortly before the TFTs are rendered conducting (by applying $V_{\mathrm{TFT}-o n}$ ) and ends shortly before the TFTs are rendered nonconducting (by applying $\left.V_{\text {TFT-off }}\right)$. Alternatively, charge integration can be terminated shortly after the TFTs are made nonconducting (method B). Thus, the size of the switching transient presented to the preamplifier, associated with rendering the TFTs nonconducting, can be obtained by subtracting the method A measurement from that of method B. The magnitude of the transient thereby obtained was measured as a function of $V_{\text {TFT-on }}$.

\section{Temporal behavior of pixel sampling}

The minimum time required to sample (and simultaneously reinitialize) a row of pixels during readout of an array determines both the maximum frame rate in fluoroscopic applications as well as the minimum time for image acquisition in radiographic applications. As the length of the gate lines and the number of pixels along these lines grow with increases in array size and pixel density, and as the width of the lines decreases to allow higher fill factors, the resistance and capacitance along the gate line could become sufficiently large so as to increase the time required to sample the pixels beyond that predicted by the simple exponential behavior given in Eq. (4). ${ }^{34}$ The large size and high pixel density of the present arrays make it inherently interesting to examine and quantify the temporal behavior of pixel sampling in order to determine the maximum frame rate that this behavior implies. With the pixel TFTs kept conducting for a fixed duration (Table II), this temporal behavior was examined by allowing pixel charge to be integrated in the preamplifiers for periods ranging from $\sim 1$ to $120 \mu$ s. (In order to ensure that the intrinsic temporal behavior associated with the preamplification circuitry itself did not affect the measurement, a preamplifier design, slightly different from that used in the other measurements, with an appropriately short time constant was employed.) These measurements were performed as a function of $V_{\mathrm{TFT}-\mathrm{on}}$.

\section{E. Relative pixel efficiency}

In order to maintain the good efficiency for detection of light photons illustrated in Fig. 2, the electric field across the photodiode (and hence the externally applied photodiode re- 
verse bias voltage) must be kept sufficiently high to maintain full depletion across the intrinsic layer. Given the various considerations which govern the choice of $V_{\text {bias }}$ (including those in Sec. II C), a knowledge of the dependence of pixel signal size on this operational parameter is crucial to ensuring optimal operation of the imager. Since the absolute light detection efficiency of the photodiode is already known, this investigation focused on an examination of how the relative pixel efficiency changed with $V_{\text {bias }}$. This was accomplished by measuring the size of the pixel signal as a function of $V_{\text {bias }}$ for a constant light signal. Furthermore, these studies were conducted for fluoroscopic and radiographic modes and for a pair of typical values for $V_{\text {TFT-on }}$. The amount of light provided per frame by the LED was constant for all measurements and was chosen so as to ensure that the signal size never exceeded $50 \%$ of pixel saturation. For the reported pixel signal results, the dark signal component, acquired in the absence of light but under otherwise identical conditions, has been subtracted. (This dark component constitutes an offset due to the combined effect of the acquisition electronics, the pixel switching transient, and TFT and photodiode dark currents.) The resulting (dark-subtracted) pixel signal is denoted by $Q_{\text {pix }}$.

\section{F. Pixel response and linearity}

An examination of a plot of the pixel signal, $Q_{\text {pix }}$, as a function of the incident radiation signal, called the pixel response curve, can clearly illustrate the influence of operational parameters and underlying phenomena on pixel performance. Pixel response curves were obtained under both fluoroscopic and radiographic conditions for a range of $V_{\text {bias }}$ values. The curves were measured as a function of increasing light signal up to saturation.

While the pixel response inevitably deviates from linearity as saturation is approached, charge trapping may cause such deviation to occur significantly before saturation. ${ }^{32,33} \mathrm{~A}$ quantitative examination of the shape of a pixel response curve allows a determination of the range over which the response of the pixel remains linear as well as a comparative assessment of the effects of mode and $V_{\text {bias }}$. The technique used in this investigation is similar to that used in previous studies. ${ }^{32,33}$ For each pixel response curve, a separate linear fit is performed to the initial part of the curve up to $\sim 20 \%$ of pixel saturation. Then for each point along the curve up to saturation, the value given by the linear fit minus the measured signal is determined. This difference, expressed as a percentage of the saturation signal size, gives a measure of the deviation of the response from linearity.

\section{G. Pixel charge trapping and release}

The trapping and release of charge from deep metastable states in the photodiode affect many of the signal and noise properties of the pixels. For this reason, it is useful and interesting to examine the magnitude and systematics of charge trapping and release. The techniques used in this investigation involved a combination of measurements in the dark, in fluoroscopic mode, and in radiographic mode.
In order to quantify the effects of charge trapping, data from pixel response curves obtained using radiographic mode were acquired as a function of $V_{\text {bias }}, T_{\text {rad-init }}$, and incident light signal up to saturation. In addition, data from pixel response curves obtained using fluoroscopic mode were also acquired as a function of $V_{\text {bias }}$ and light signal. (The data used in this investigation included the fluoroscopic and radiographic pixel response curves generated for the studies described in Sec. III F. For both modes, $T_{\text {frame }}$ was fixed at $33 \mathrm{~ms}$.) For a given value of $V_{\text {bias }}$ and $T_{\text {rad-init }}$, the (darksubtracted) radiographic pixel signal was subtracted from the (dark-subtracted) fluoroscopic pixel signal obtained at the same light level. While the radiographic pixel signal is reduced due to charge trapping, the corresponding fluoroscopic pixel signal is acquired under conditions of equilibrium between charge trapping and release. Thus, the difference between the two signals, $Q_{\text {trap }}$, represents the magnitude of charge trapping relative to conditions of equilibrium. Finally, the reported $Q_{\text {trap }}$ results are expressed as a percentage of the corresponding (dark-subtracted) fluoroscopic pixel signal level.

In order to quantify the effects of charge release, data were acquired using a methodology involving a combination of fluoroscopic pixel response curve measurements plus an additional dark measurement. For a given value of $V_{\text {bias }}$, $T_{\text {frame }}$, and light level, pixel signal size in fluoroscopic mode was determined from an average of the final image frames in a consecutive sequence, as described at the beginning of this section. In addition, pixel data were acquired from the first frame following the last image frame of this sequence (i.e., the first frame for which the LED was not flashed). When the dark signal component is subtracted from the signal from this additional frame, the remaining pixel signal, $Q_{\text {release }}$ represents the release of charge trapped during previous frames. $Q_{\text {release }}$ was measured as a function of $V_{\text {bias }}, T_{\text {frame }}$, and incident light signal up to saturation. As in the case of $Q_{\text {trap }}$, the presented $Q_{\text {release }}$ results are expressed as a percentage of the corresponding (dark-subtracted) fluoroscopic pixel signal size in order to quantify the effect relative to conditions of equilibrium.

\section{H. X-ray sensitivity}

It is intrinsically interesting and important to measure the size of the pixel signal per unit $\mathrm{x}$-ray exposure as this is a fundamental characteristic of an imager and such information is necessary for a signal-to-noise analysis of the system. This quantity, which we refer to as x-ray sensitivity, was examined as a function of beam energy and screen type for both fluoroscopic and radiographic modes with a single set of operational parameters. Data were obtained from 70 to $120 \mathrm{kVp}$ for the three commercially available screens mentioned in Sec. II A. As there was no phantom or other material placed between the source and the imager, the resulting sensitivities correspond to the unattenuated beam. For a given beam energy, screen type, and mode, the x-ray sensitivity was given by the slope determined from a linear regression of pixel signal measurements versus exposure. In 
order to assure that the sensitivities were determined under conditions where the pixel response was highly linear, pixel signal data were acquired for pixel signals not exceeding $\sim 20 \%$ of saturation. The exposure at the array surface was measured by means of a Keithley ion chamber, model 95035 , used with a Keithley 35050 A digital dosimeter. Given the very limited control of exposure rate allowed by the x-ray source in radiographic mode, the large differences in optical gain between the three screens, and the requirement that the pixel signal not exceed $\sim 20 \%$ of saturation, it proved necessary to locate the surface of the imager progressively farther from the x-ray source for higher gain screens. Consequently, for the fluoroscopic and radiographic measurements, the source to imager distance involving Lanex Fine, Regular, and Fast-B screens was 65, 80, and $123 \mathrm{~cm}$, respectively.

\section{X-ray images}

In order to provide visual examples of the performance of the imager, x-ray images of a variety of objects were acquired. A qualitative illustration of the spatial resolution of the system was provided by images of a spatial resolution, 0.05-mm-thick, lead line-pair pattern (model 88548; Nuclear Associates). These images were acquired both fluoroscopically and radiographically using the high-resolution Lanex Fine screen. The source-to-detector distance was $100 \mathrm{~cm}$ and the pattern was placed in direct contact with the screen. Using the maximum pixel block which could be addressed by the present acquisition system $(512 \times 1920$ pixels), an $x$-ray image of the hand of one of the investigators was acquired radiographically. In order to demonstrate images using the full surface of the array, images were also acquired of an anthropomorphic hand phantom both fluoroscopically and radiographically. As only one-third of the array data lines (i.e., 512) could be addressed at a time, each of these images was produced by combining image frames acquired separately from each third of the array. All hand images were obtained with the imager at $\sim 86 \mathrm{~cm}$ from the $\mathrm{x}$-ray source using the Lanex Regular screen. The choice of this lowerresolution, higher-gain screen (compared to the Lanex Fine) was driven by the desire to minimize the exposure while maintaining the pixel signals at a level where the preamplifier noise did not significantly degrade the images. The only image processing performed to the images consisted of the application of a gain and offset correction to each pixel as well as the use of a median filter to partially remove the distracting influence of pixel and line defects. ${ }^{2,35}$

\section{RESULTS}

The results reported in this section correspond to single pixel measurements and array images acquired in the manner outlined in Sec. III. For each property studied, data were obtained for a number of pixels in the selected pixel block and representative results, usually from a single pixel, are shown in the following figures. Except where noted, all results correspond to an array incorporating the new oxynitride

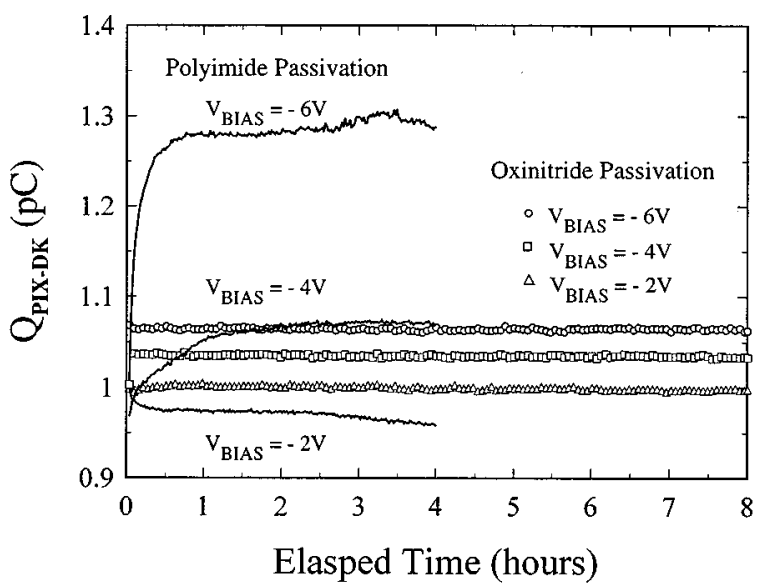

FIG. 4. Plot of pixel dark signal as a function of elapsed time illustrating the temporal stability of this property. For photodiode reverse bias voltages, $V_{\text {bias }}$, of $-2,-4$, and $-6 \mathrm{~V}$, data are shown both for a pixel from an array with an oxynitride passivation layer (triangles, squares, and circles, respectively) as well as for a pixel from an array with a polyimide passivation (solid lines). Note that for this plot, and for that shown in Fig. 5(a), the absolute magnitude of the signal contains an unknown offset. Finally, the polyimide results have been renormalized so that at the shortest elapsed time at $-2 \mathrm{~V}$, the polyimide and oxynitride data are equal.

passivation layer. For purposes of comparison, a small amount of data are shown for an array of identical design but incorporating a polyimide passivation layer.

\section{A. Pixel dark signal drift}

The behavior of the pixel dark signal over $4-8 \mathrm{~h}$ of continuous imager operation is shown in Fig. 4. Data are shown for a single pixel from the array with the oxynitride passivation layer as well as from the polyimide array. For each array, data are shown for values of $V_{\text {bias }}$ approximately spanning the practical range. While the magnitude of the presented results for a given array contains an unknown offset (as explained in Sec. III A), the vertical scale correctly describes changes over time as well as differences for different values of $V_{\text {bias }}$. The oxynitride array demonstrates extremely little signal drift (less than $0.01 \mathrm{pC}$ ) over an $8 \mathrm{~h}$ period, aside from fluctuations during the first few minutes after the voltage is initially applied. Second, for an increase in $V_{\text {bias }}$ from -2 to $-6 \mathrm{~V}$ the increase in dark signal was only $\sim 0.07 \mathrm{pC}$. This represents a very small fraction of the calculated pixel signal capacity of $\sim 4.2 \mathrm{pC}$ at $-6 \mathrm{~V}$ [from Eq. (2) using the measured photodiode capacitance of $0.7 \mathrm{pF}$ (Sec. IV F)]. By comparison, the polyimide array shows considerable drift (up to $0.3 \mathrm{pC}$ at $-6 \mathrm{~V}$ ) and a far larger increase (up to $\sim 0.35$ $\mathrm{pC}$ ) in dark signal from -2 to $-6 \mathrm{~V}$.

\section{B. Pixel dark signal}

The variation of the pixel dark signal with frame time for the oxynitride and polyimide arrays is shown in Fig. 5(a). Once again, for a given array, while the magnitude of the signal contains an unknown offset, the vertical scale correctly describes changes with $V_{\text {bias }}$ and $T_{\text {frame }}$. In order to minimize the influence of dark current drift (see Fig. 4), 

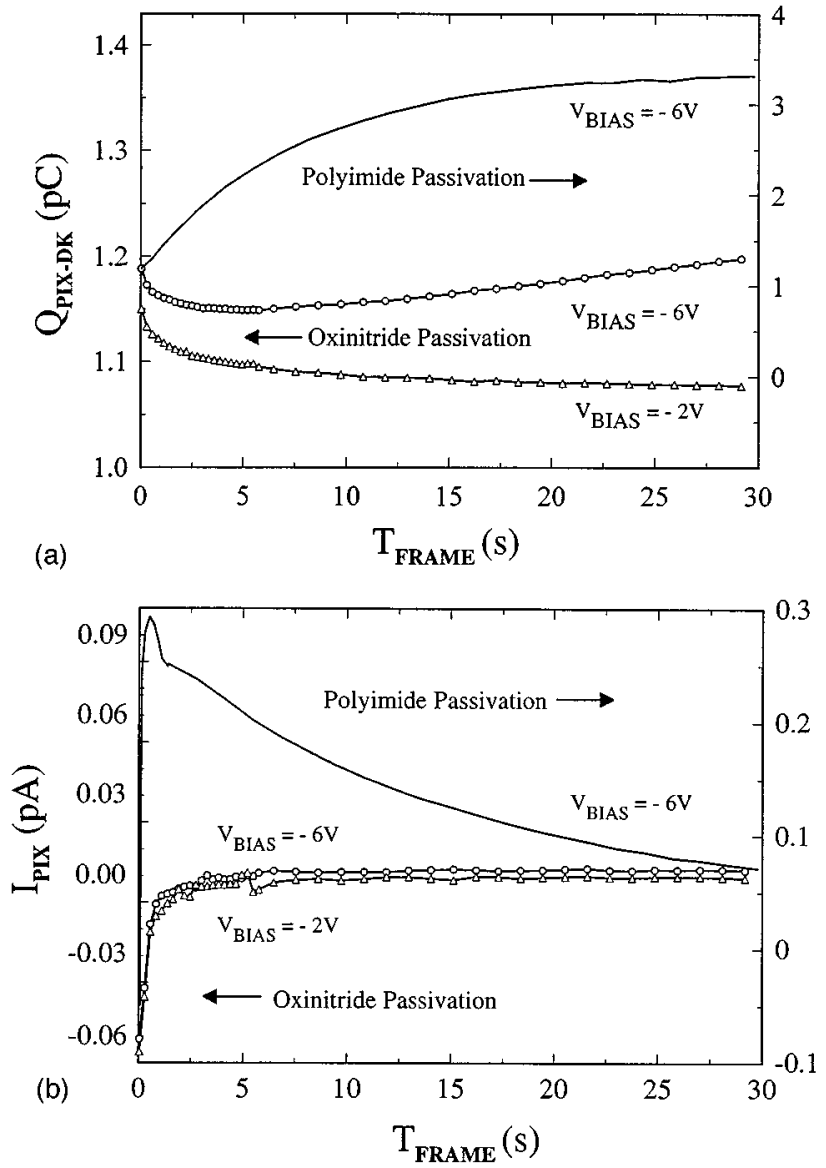

FIG. 5. (a) Plot of pixel dark signal as a function of frame time, $T_{\text {frame }}$, for the oxynitride array at $V_{\text {bias }}$ values of -2 and $-6 \mathrm{~V}$ and for the polyimide array at $-6 \mathrm{~V}$. Through the application of an additive offset, the polyimide results have been renormalized to coincide with the $-6 \mathrm{~V}$ oxynitride data at the shortest frame time. (b) Plot of the pixel dark current as a function of $T_{\text {frame }}$ derived from the Fig. 5(a) data. For both plots, the oxynitride data correspond to the open triangles and circles and the left vertical scale while the polyimide data correspond to the solid line and the right vertical scale.

measurements for a given $V_{\text {bias }}$ were performed only after the array was continually read out for 1 and $3 \mathrm{~h}$ for the oxynitride and polyimide arrays, respectively. For the oxynitride array, at $-2 \mathrm{~V}$ the dark signal shows a steady decay toward an asymptotic limit. At $-6 \mathrm{~V}$ there is an initial decay up to a $T_{\text {frame }}$ of $\sim 5 \mathrm{~s}$ followed by a slow, linear increase in dark signal. This behavior most likely reflects the competing effects of the TFT transient current (which apparently dominates at $-2 \mathrm{~V}$ ) and the photodiode dark current (which is larger at $-6 \mathrm{~V}$ and which eventually dominates over the decaying TFT transient current). In the case of the polyimide array, photodiode dark current is considerably greater and thus the dark signal exhibits a large, monotonic increase (representing a substantial fraction of the pixel signal capacity) before appearing to approach an asymptotic limit at large values of $T_{\text {frame }}$. Overall, the polyimide array exhibits changes in dark signal with $T_{\text {frame }}$ up to $\sim 36$ times larger than for the oxinitride array at $-6 \mathrm{~V}$.

Using the data of Fig. 5(a), the absolute magnitude of the pixel dark current was determined and the results are plotted

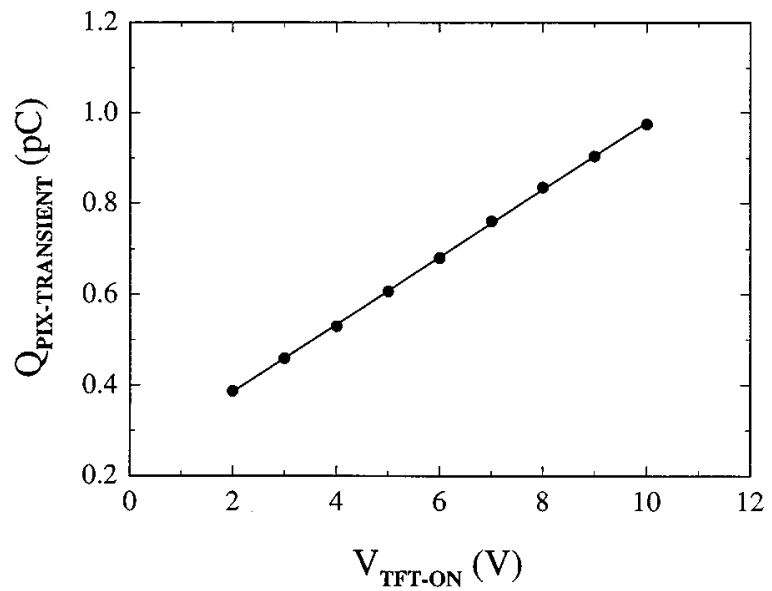

FIG. 6. Plot of the pixel switching transient presented to the preamplifier as a function of TFT-on voltage, $V_{\text {TFT-on }}$. Data are shown for values of $V_{\text {TFT-on }}$ ranging from $2 \mathrm{~V}$, a value slightly above the TFT threshold voltage, to $10 \mathrm{~V}$. The line represents a least-squares fit to the data.

in Fig. 5(b). For the oxynitride array, the dark current changes from a value of -0.065 and $-0.061 \mathrm{pA}$ at $T_{\text {frame }}=35 \mathrm{~ms}$ to -0.013 and $-0.008 \mathrm{pA}$ at $T_{\text {frame }}=1.11 \mathrm{~s}$ for $V_{\text {bias }}=-2$ and $-6 \mathrm{~V}$, respectively. Thereafter, the dark current increases only very slowly up to values of -0.0008 and $0.0020 \mathrm{pA}$ at $\sim 29 \mathrm{~s}$, respectively. In the case of the polyimide array, much larger dark current and dark current variation is observed. While the polyimide dark current is comparable to that of oxynitride at very short frame times $(-0.050 \mathrm{pA}$ at $74 \mathrm{~ms}$ ), it quickly increases to $0.29 \mathrm{pA}$ at $0.50 \mathrm{~s}$ before gradually decreasing to $0.07 \mathrm{pA}$ at $\sim 30 \mathrm{~s}$. At $T_{\text {frame }}=150 \mathrm{~ms}$, $1 \mathrm{~s}$, and $\sim 30 \mathrm{~s}$, the magnitude of the dark current for the polyimide array is about $2.7,33$, and 29 times larger than for the oxynitride array.

\section{Pixel switching transient}

The magnitude of the switching transient presented to the preamplifier when the gate line voltage is switched from $V_{\text {TFT-on }}$ to $V_{\text {TFT-off }}$ is plotted as a function of $V_{\text {TFT-on }}$ in Fig. 6 . The data exhibit a linear dependence upon $V_{\text {TFT-on }}$. At values of $V_{\text {TFT-on }}$ closer to the threshold voltage $(\sim 1 \mathrm{~V})$, the behavior of the TFT is highly nonlinear and practical operation of the array is precluded. Perhaps the most interesting aspect of these data is the magnitude of the transient which ranges from 0.4 to $\sim 1 \mathrm{pC}$ for $V_{\text {TFT-on }}=2$ to $10 \mathrm{~V}$, respectively. This is found to agree with theoretical estimates to within a factor of 2. Given that a switching transient of comparable size is believed to be injected into the photodiode when the TFT is turned off, and since this signal represents a large fraction of the pixel signal capacity which ranges from $\sim 1.4$ to $\sim 4.2 \mathrm{pC}$ for $V_{\text {bias }}=-2$ to $-6 \mathrm{~V}$, respectively, it is expected that the effect of this charge injection should be manifest in the operation of the pixels. This is indeed the case as reported in Sec. IV E. Finally, it is interesting that, as a result of design changes incorporated into the array, including those outlined 


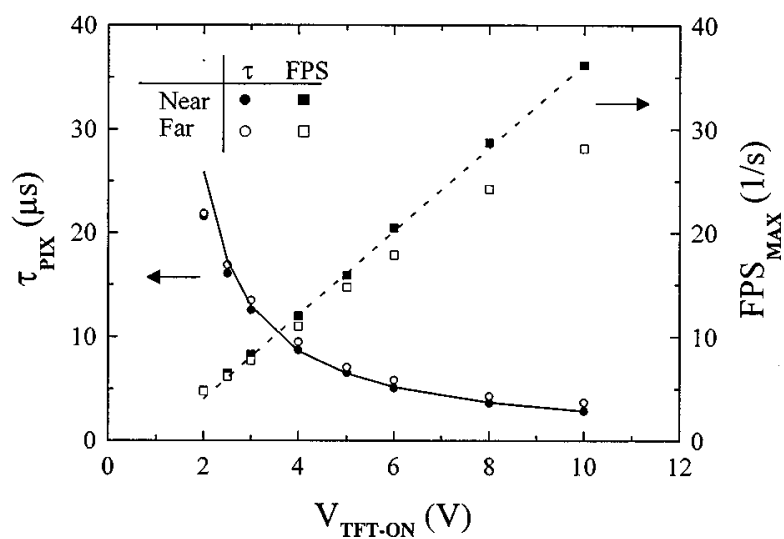

FIG. 7. Measurements and calculations for pixels near to (solid symbols) and far from (open symbols) gate driver circuitry. Left-hand scale: plot of pixel time constant, $\tau_{\text {pix }}$, vs $V_{\text {TFT-on }}$. The solid line is the result of a fit to the near pixel data. Right-hand scale: Plot of the maximum theoretical frame rates implied by the measured time constants. The dashed line represents frame rates corresponding to the time constants represented by the solid line.

in Sec. II A, the switching transient for the present array has been reduced by over a factor of 6 compared to that of earlier arrays with a $450 \mu \mathrm{m}$ pitch.

\section{Temporal behavior of pixel sampling}

Using the technique described in Sec. III D, pixel charge was sampled as a function of preamplifier integration time for pixels at both ends of the gate lines. In this way, data were gathered both for pixels very close to the gate driving circuitry and maximally distant from this circuitry. All the data were found to exhibit the exponential behavior described by Eq. (4). Each set of data was fit with a function of the form of Eq. (4) to determine a pixel time constant, $\tau_{\text {pix }}$. The values of $\tau_{\text {pix }}$ thereby measured are plotted as a function of $V_{\text {TFT-on }}$ in Fig. 7. The far pixel data exhibit a slightly larger time constant than the near pixel data, which is most likely a result of the increased resistive and capacitive load between the drivers and the far pixels. The solid line corresponds to a fit of the near pixel data using Eqs. (5) and (3). In this fit, the photodiode capacitance, $C_{\mathrm{pd}}$, was fixed at the empirically determined value of $0.7 \mathrm{pF}$ (see Sec. IV F) while the field effect mobility, $\mu_{\mathrm{fe}}$ and TFT threshold voltage, $V_{T}$, were allowed to vary. Except at values of $V_{\text {TFT-on }}$ near threshold where the response of the transistor is not as precisely described by Eq. (3), the near pixel data are well fit using typical values for $\mu_{\mathrm{fe}}$ and $V_{T}\left(0.75 \mathrm{~cm}^{2} / \mathrm{V} \mathrm{s}\right.$ and $1.0 \mathrm{~V}$, respectively-Table II). Using these empirically determined values of $\tau_{\text {pix }}$, the maximum theoretical frame rate for the entire $127 \mu \mathrm{m}$ array may be calculated using Eq. (6b) and the results are also plotted in Fig. 7. These calculations indicate that, despite the reduced time constants of the far pixels, the properties of the array allow readout at $\sim 30 \mathrm{fps}$ under typical operational conditions.

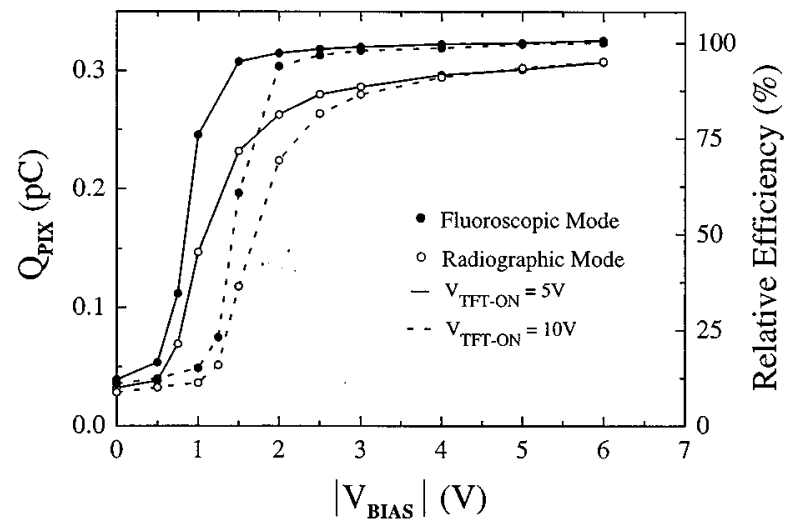

FIG. 8. Plot illustrating the relative efficiency of light detection by a pixel. Dark-subtracted pixel signal, $Q_{\text {pix }}$, is plotted as a function of the magnitude of the photodiode reverse bias voltage for fluoroscopic mode and radiographic mode. Data are shown for TFT-on voltages, $V_{\mathrm{TFT}-\text { on }}$, of 5 and $10 \mathrm{~V}$ as indicated by the connecting solid and dashed lines, respectively. $T_{\text {rad-init }}$ and $T_{\text {frame }}$ were fixed at $10 \mathrm{~s}$ and $33 \mathrm{~ms}$, respectively. The left vertical scale gives the pixel signal size in units of charge while the right vertical scale gives the signal size expressed as a percentage of the asymptotic fluoroscopic signal size at $V_{\text {bias }}=-6 \mathrm{~V}$.

\section{E. Relative pixel efficiency}

The measurements of relative pixel efficiency as a function of $V_{\text {bias }}, V_{\text {TFT-on }}$, and mode reveal an interesting variety of behaviors. These are illustrated in Fig. 8 which shows plots of (dark-subtracted) pixel signal versus $V_{\text {bias }}$. For each measurement set, the pixel exhibits only a limited response at low $V_{\text {bias }}$, rises steeply starting from $\sim-0.5$ to $\sim-1.0 \mathrm{~V}$, and nears its asymptotic value at $\sim-1.5$ to $\sim-3.0 \mathrm{~V}$. For each mode, the relative efficiency curve for $V_{\text {TFT-on }}=10 \mathrm{~V}$ is shifted $\sim 0.6 \mathrm{~V}$ relative to the $V_{\text {TFT-on }}=5 \mathrm{~V}$ curve as measured by comparison of the $50 \%$ points between curves. In addition, the radiographic curves exhibit a slower increase with $V_{\text {bias }}$ and asymptotically approach a slightly lower signal level than the fluoroscopic results. Finally, while the results shown in Fig. 8 correspond to specific values for $T_{\text {frame }}$ and $T_{\text {rad-init }}$, no significant variation from the presented results were observed when these parameters were varied over the ranges given in Table IV.

The behavior observed in Fig. 8 in fluoroscopic mode for $V_{\text {TFT-on }}=10 \mathrm{~V}$ is distinctly different from that from an earlier array $(512 \times 560$ pixels, $450 \mu \mathrm{m}$ pitch) having considerably larger photodiodes and pixel signal capacity. ${ }^{33}$ For the earlier array, the relative efficiency increased very steeply from $0 \mathrm{~V}$ and neared its maximum at $\sim-0.5 \mathrm{~V}$, i.e., the data exhibited only a $\sim 0.25 \mathrm{~V}$ shift relative to $V_{\text {bias }}=0 \mathrm{~V}$. The present array exhibits much larger $V_{\text {bias }}$ shifts due to the comparatively large size of the pixel switching transient relative to the pixel signal capacity. Interpreting these shifts as the result of injected charge and given the measured photodiode capacitance of $\sim 0.7 \mathrm{pF}$, the $\sim 0.8$ and $\sim 1.4 \mathrm{~V}$ shifts in the $V_{\text {TFT-on }}=5$ and $10 \mathrm{~V}$ fluoroscopic curves with respect to $0 \mathrm{~V}$ imply an injected charge of $\sim 0.6$ and $\sim 1 \mathrm{pC}$, respectively. This is in good agreement with the measurements of charge injected into the preamplifier (Fig. 6). Thus, data such as 


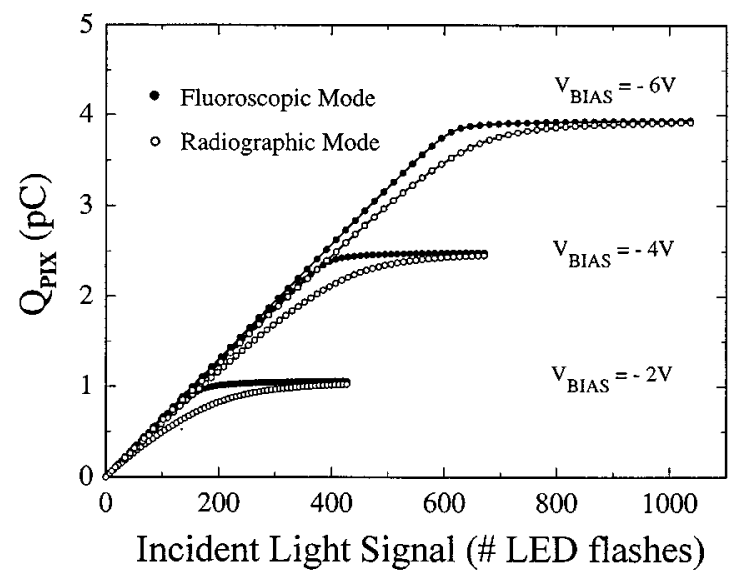

FIG. 9. Dark-subtracted pixel signal, $Q_{\text {pix }}$, plotted as a function of incident light signal, in units of number of LED flashes. Data are presented for $V_{\text {bias }}$ values of $-2,-4$, and $-6 \mathrm{~V}$ for both fluoroscopic and radiographic modes. $T_{\text {rad-init }}$ and $T_{\text {frame }}$ were fixed at $10 \mathrm{~s}$ and $33 \mathrm{~ms}$, respectively.

those shown in Fig. 8 illustrate the effect of the switching transient and quantify both the reduction in the effective reverse bias voltage applied across the photodiodes and the corresponding reduction in relative pixel efficiency.

Moreover, the slower increase in relative efficiency and the lower efficiency ultimately achieved in the radiographic results of Fig. 8 may be attributed to the effect of charge trapping. Whereas the fluoroscopic measurements are made under conditions where equilibrium is established between the trapping and release of charge, such equilibrium is not present during the radiographic measurements. Thus, there is a net loss of signal due to trapped charge affecting both the shape and asymptotic magnitude of the relative efficiency curves.

\section{F. Pixel response and linearity}

Pixel signal as a function of incident light signal is plotted in Fig. 9 for a range of $V_{\text {bias }}$ values for both fluoroscopic and radiographic mode. Each pixel response curve initially exhibits highly linear behavior before progressively deviating from linearity and saturating. The saturation levels are found to increase linearly with $V_{\text {bias }}$ as per Eq. (2). With Eq. (2) and using saturation data including those shown in Fig. 9, an empirical determination of the photodiode capacitance, $C_{\mathrm{pd}}$, yields a value of $0.7 \mathrm{pF}$. This implies a value of $\sim 1.0 \mu \mathrm{m}$ for the nominal thickness for the intrinsic layer of the photodiode [using Eq. (1)], which is consistent with the range for this parameter due to processing variations during array fabrication.

It is particularly instructive to compare pixel response curves obtained using fluoroscopic and radiographic modes. As illustrated in Fig. 9, for a given value of $V_{\text {bias }}$, the response of the imager in radiographic mode is lower than in fluoroscopic mode. Initially, as the incident light signal increases, the absolute difference in response between the two modes systematically increases. However, as pixel saturation is approached, the size of the pixel signal becomes con-
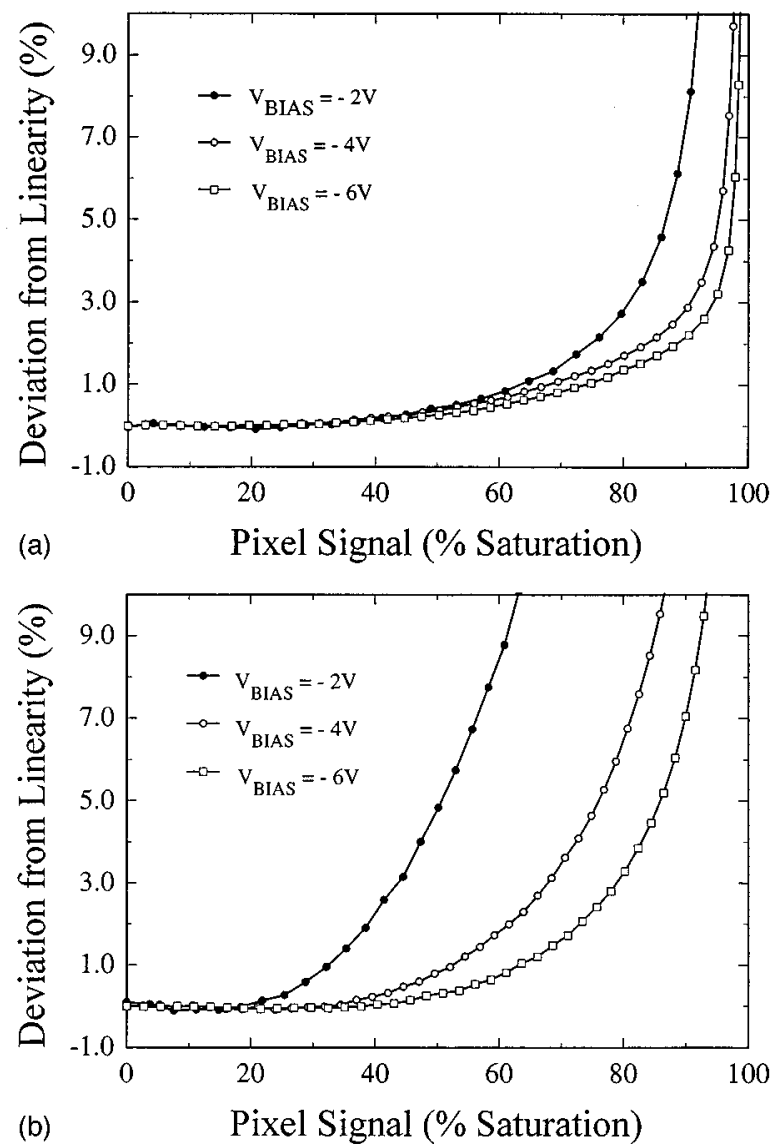

FIG. 10. Plot of deviation from linearity as a function of pixel signal size for (a) fluoroscopic and (b) radiographic modes, respectively. The results shown correspond to an analysis of the data shown in Fig. 9. Note that positive values of deviation correspond to a sublinear pixel response. See the text for details.

strained and the fluoroscopic and radiographic mode data converge. As in the case of the relative pixel efficiency measurements, these differences may be attributed to the effect of charge trapping in radiographic mode which, unlike fluoroscopic mode, is not compensated for by charge release.

While the fluoroscopic results shown in Fig. 9 were obtained with $T_{\text {frame }}$ fixed, additional investigations indicated that for a given light level, the fluoroscopic signal size was relatively independent of frame time over at least the range of values given in Table IV. For example, for $V_{\text {bias }}=-2 \mathrm{~V}$ $(-6 \mathrm{~V})$, no variation with $T_{\text {frame }}$ was observed up to $\sim 60 \%$ $(\sim 80 \%)$ of saturation and deviations of only $\sim 5 \%(1 \%)$ at $\sim 95 \%$ of saturation were observed. Moreover, for a given light level and a fixed value of $T_{\text {rad-init }}$, the radiographic signal size was found to be independent of frame time for values of $T_{\text {frame }}$ ranging from $33 \mathrm{~ms}$ to $1 \mathrm{~s}$. However, radiographic signal size was found to decrease with increasing $T_{\text {rad-init }}$ as reported in Sec. IV G.

Following the method outlined in Sec. III F, an analysis of the pixel response data of Fig. 9 yields the results shown in Figs. 10(a) and 10(b) for fluoroscopic and radiographic modes, respectively. In these figures, the deviation of pixel response from linearity is plotted as a function of the size of 


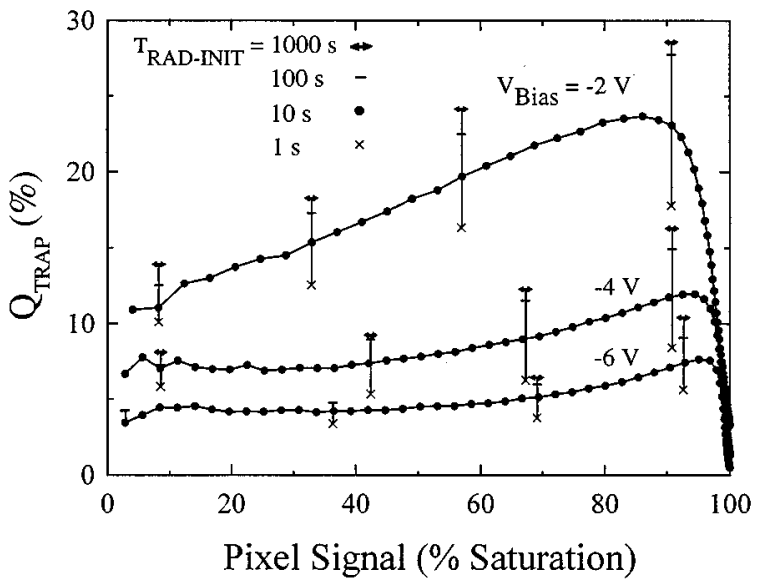

FIG. 11. Trapped charge, $Q_{\text {trap }}$, plotted as a function of fluoroscopic pixel signal size for $V_{\text {bias }}$ values of $-2,-4$, and $-6 \mathrm{~V}$. $Q_{\text {trap }}$ is the amount of missing charge from a radiographic mode measurement relative to the size of the pixel signal under conditions of equilibrium between charge trapping and charge release in fluoroscopic mode-see the main text for details. The horizontal scale represents the size of the corresponding fluoroscopic pixel signal size at equilibrium relative to saturation. The solid circles represent data points acquired with a radiographic initialization time, $T_{\text {rad-init }}$, of $10 \mathrm{~s}$. For each set of data at a particular $V_{\text {bias }}$, additional data illustrating the amount of trapping at $T_{\text {rad-init }}$ values of 1,100 , and $1000 \mathrm{~s}$ are indicated by crosses, horizontal bars, and double arrows, respectively.

the pixel signal, expressed as a percentage of saturation. In both modes, the deviation from linearity increases with increasing signal size and decreasing $V_{\text {bias }}$ as a result of decreasing electric field across the photodiode. Perhaps the most notable aspect of these data is the degree to which the behavior of the imager in fluoroscopic mode differs from that in radiographic mode. Generally, the fluoroscopic data demonstrate a greater range of highly linear behavior than the radiographic data. For example, whereas the fluoroscopic data exhibit deviations from linearity below the $1 \%$ level up to $\sim 64 \%$ to $\sim 75 \%$ of the signal range for $V_{\text {bias }}=-2$ to -6 $\mathrm{V}$, respectively, the radiographic data exhibit such variations only up to $\sim 32 \%$ to $\sim 63 \%$. As previously mentioned, it is likely that this is a manifestation of a net charge trapping effect in radiographic mode. On the other hand, once the response starts to deviate from linearity, the rate at which it does so with increasing signal size and $V_{\text {bias }}$ appears to be greater in the case of fluoroscopic mode. Since fluoroscopic mode does not begin to exhibit significant deviation from linearity until the pixel signals are much closer to saturation, this enhanced rate of deviation may simply be the result of more sharply decreasing pixel efficiency as saturation is approached and the effective voltage bias across the photodiode diminishes (see Sec. IV E and Fig. 8).

\section{G. Pixel charge trapping and release}

In Fig. 11, charge trapping in plotted as a function of pixel signal for a range of $V_{\text {bias }}$ values. As discussed in Sec. II C 4, it is generally anticipated that the trapping of charge should increase with decreasing sensor bias voltage and/or increasing pixel signal size. This expectation is generally

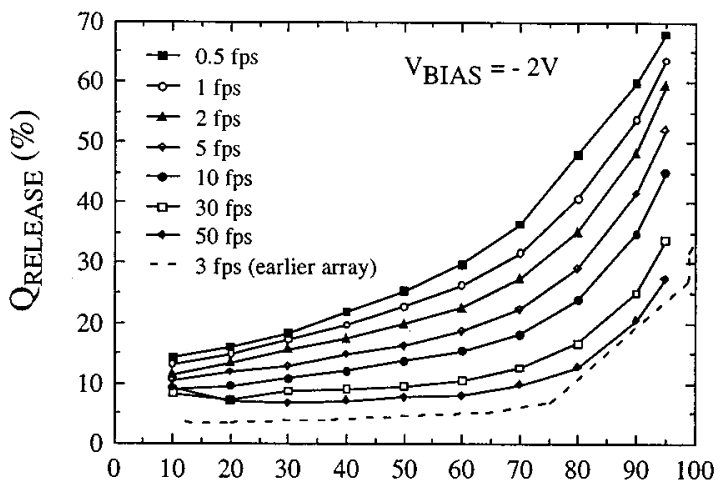

(a)
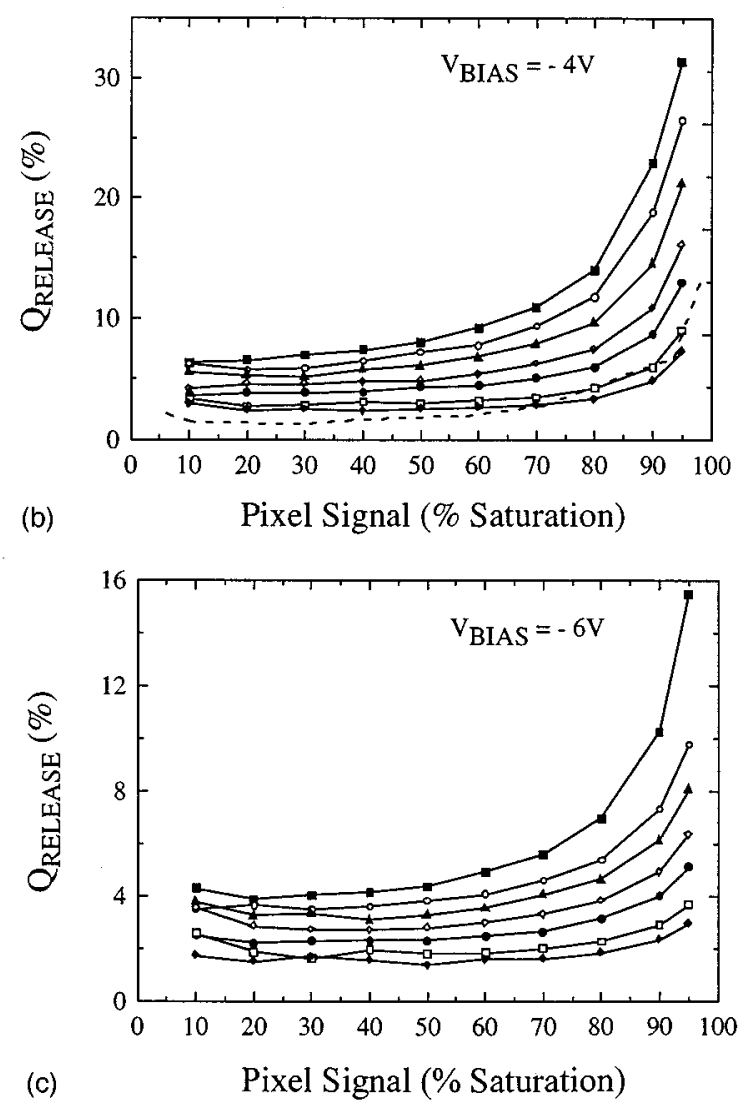

FIG. 12. Released charge, $Q_{\text {release }}$, plotted as a function of fluoroscopic pixel signal size for $V_{\text {bias }}$ values of (a) $-2 \mathrm{~V}$, (b) $-4 \mathrm{~V}$, and (c) $-6 \mathrm{~V}$. $Q_{\text {release }}$ is the amount of charge released in the first frame following a series of fluoroscopic image frames-see the main text for details. For each value of $V_{\text {bias }}$, data were acquired for seven values of $T_{\text {frame }}(20,33,100,200,500$, 1000 , and $2000 \mathrm{~ms}$ ). These data are labeled in the figure in terms of the corresponding frame rate as given by the inverse of $T_{\text {frame }}$. The convention for the horizontal scale is the same as for Fig. 11. For each plot, results for individual frame rates are joined by solid lines for reasons of clarity. For purposes of comparison, the dashed line superimposed on Figs. 12(a) and 12(b) represents previously reported results from a $64 \times 40$ pixel, $900 \mu \mathrm{m}$ pitch array operated at $\sim 3 \mathrm{fps}$ (Ref. 36).

borne out in the $Q_{\text {trap }}$ data shown in Fig. 11. The dependence on pixel signal size is particularly evident at $V_{\text {bias }}=-2 \mathrm{~V}$. (The apparently anomalous decrease in $Q_{\text {trap }}$ with increasing pixel signal observed near saturation is simply an artifact of the methodology ${ }^{36}$ - the size of the average pixel signal be- 
TABLE V. Sensitivity measurements, in units of $\mathrm{pC} / \mathrm{mR}$, obtained from individual imaging pixels. Results are shown for fluoroscopic and radiographic modes for three screens.

\begin{tabular}{ccccccccc}
\hline \hline & \multicolumn{2}{c}{ Lanex Fine } & & \multicolumn{2}{c}{ Lanex Regular } & & \multicolumn{2}{c}{ Lanex Fast-B } \\
\cline { 2 - 3 } Energy $(\mathrm{kVp})$ & Fluoroscopic & Radiographic & & Fluoroscopic & Radiographic & & Fluoroscopic & Radiographic \\
\hline 70 & 0.023 & 0.023 & & 0.084 & 0.076 & & 0.163 & 0.136 \\
80 & 0.026 & 0.024 & & 0.091 & 0.081 & & 0.168 & 0.150 \\
90 & 0.027 & 0.026 & & 0.098 & 0.085 & & 0.184 & 0.163 \\
100 & 0.029 & 0.027 & & 0.102 & 0.087 & & 0.195 & 0.169 \\
110 & 0.030 & 0.027 & & 0.105 & 0.089 & & 0.203 & 0.170 \\
120 & 0.030 & 0.024 & & 0.106 & 0.091 & & 0.208 & 0.174 \\
\hline \hline
\end{tabular}

comes increasingly constrained as saturation is approached while the amount of trapped charge is not similarly constrained.) In addition, for each value of $V_{\text {bias }}$, the variation in $Q_{\text {trap }}$ over a range of values for $T_{\text {rad-init }}$ is shown (see figure caption). Generally, longer radiographic initialization times result in increased trapping, particularly at lower values of $V_{\text {bias }}$. Finally, the advantage in operating the array at large $V_{\text {bias }}($ e.g., $-6 \mathrm{~V})$ is apparent given that $Q_{\text {trap }}$ remains below $\sim 5 \%$ throughout most of the signal range.

Charge release is plotted as a function of pixel signal for a range of values of $V_{\text {bias }}$ and frame rate in Figs. 12(a)-12(c). Since the $Q_{\text {release }}$ results correspond to the charge released during only a single frame after the pixels have been brought to a charge trapping-charge release equilibrium in fluoroscopic mode, while the $Q_{\text {trap }}$ results represent missing charge for a single radiographic frame, the magnitudes of the effects shown in Figs. 11 and 12 are not expected to correspond. However, as in the case of the $Q_{\text {trap }}$ data, the amount of released charge generally decreases with increasing $V_{\text {bias }}$ and decreasing pixel size-reflecting the relative amount of trapped charge available to be released. Furthermore, the observed trend of larger $Q_{\text {release }}$ with longer $T_{\text {frame }}$ (decreasing frame rate) corresponds to the expectation that a longer frame time provides greater statistical probability for charge to be released from the trapping states. ${ }^{30}$ Finally, for purposes of comparison, results at a frame rate of $\sim 3 \mathrm{fps}$ from an earlier, more limited, study performed with a small array produced on a 4 in. reactor ${ }^{36}$ are superimposed on the -2 and $-4 \mathrm{~V}$ data of Figs. 12(a) and 12(b), respectively. Compared to the results obtained from the present array which was produced with a large-area process, these earlier results indicate a significantly lower level of charge release. Given the expectation that the quantity of released charge scales with the amount of trapped charge, it is likely that lower levels of charge trapping and release than those exhibited by the present large-area array can be realized through further improvements in the fabrication process.

\section{H. X-ray sensitivity}

Measurements of (dark-subtracted) pixel signal per unit exposure are given in Table V. Data are presented as a function of x-ray energy, phosphor screen type, and mode (fluoroscopic or radiographic). The magnitude of the radiographic results are generally smaller than the fluoroscopic results due to the effects of charge trapping discussed in the previous sections. The fluoroscopic results for the Lanex Regular and Lanex Fast-B screens show a dependence on energy and screen type similar to that observed with an earlier array $\left(560 \times 512\right.$ pixels at $450 \mu \mathrm{m}$ pitch). ${ }^{33}$ However, the magnitude of the present results, when normalized to unit optically sensitive area of the photodiode, are $\sim 10 \%-20 \%$ larger than results for the earlier array. While the reasons for this enhancement are unclear, better quantum efficiency of the photodiodes for the present array is at least partially responsible. The sensitivity data agree well with the results of theoretical calculations. $^{19}$

\section{X-ray images}

An x-ray image of a line-pair pattern acquired at $70 \mathrm{kVp}$ is shown in Fig. 13. The image was acquired in fluoroscopic mode with the long dimension of the pattern oriented approximately parallel to the data address lines of the array. Faint horizontal and vertical line artifacts due to defective gate and data address lines are evident in the image. Although such a tool is of limited use in characterizing the spatial resolution properties of a pixelated imaging system,

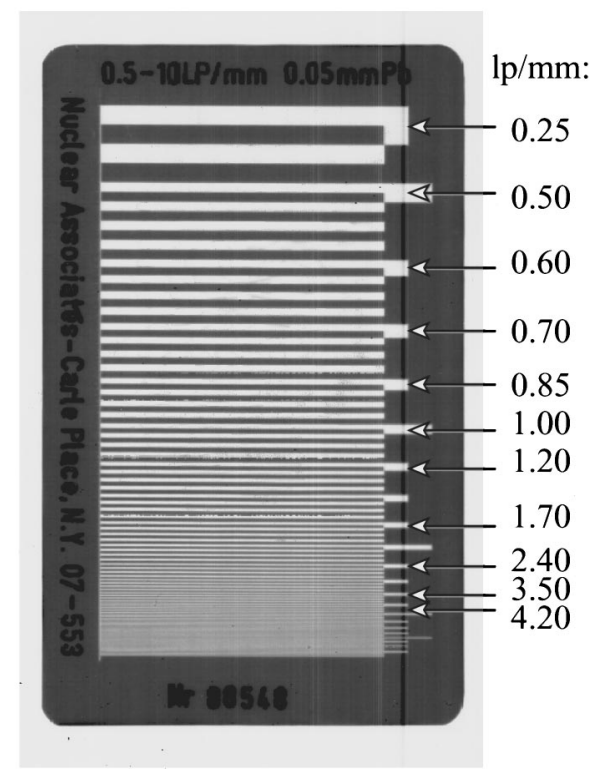

FIG. 13. X-ray image at $70 \mathrm{kVp}$ of a line-pair pattern acquired with the flat-panel imager operated in fluoroscopic mode. The manufacturer's values of $\mathrm{lp} / \mathrm{mm}$ are listed on the right-hand side. 


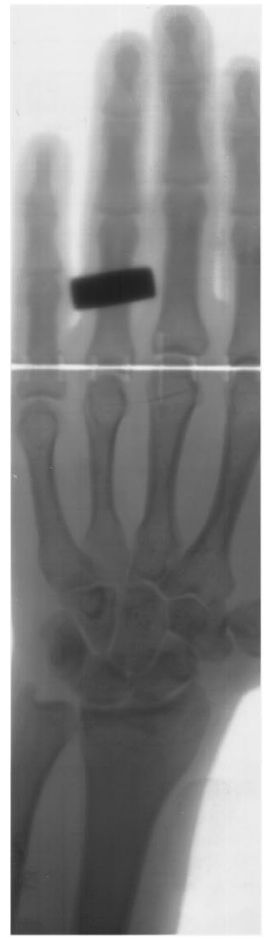

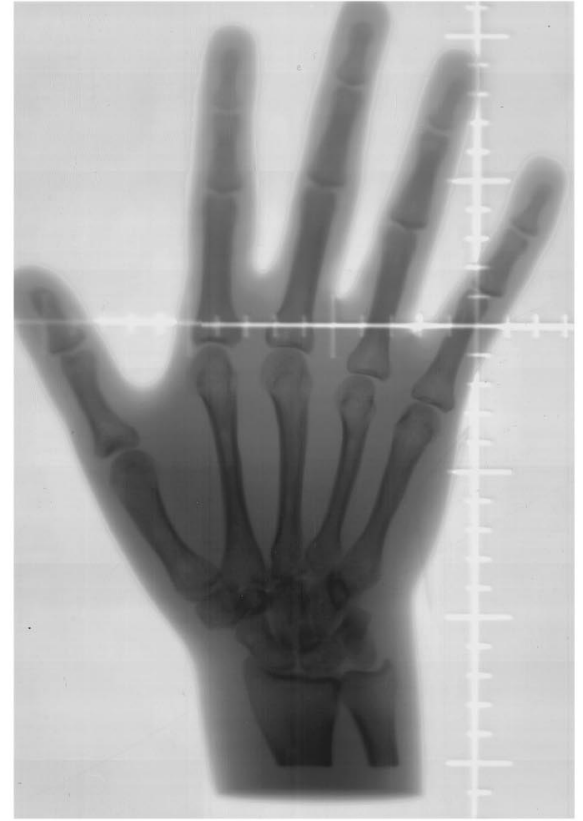

(b)

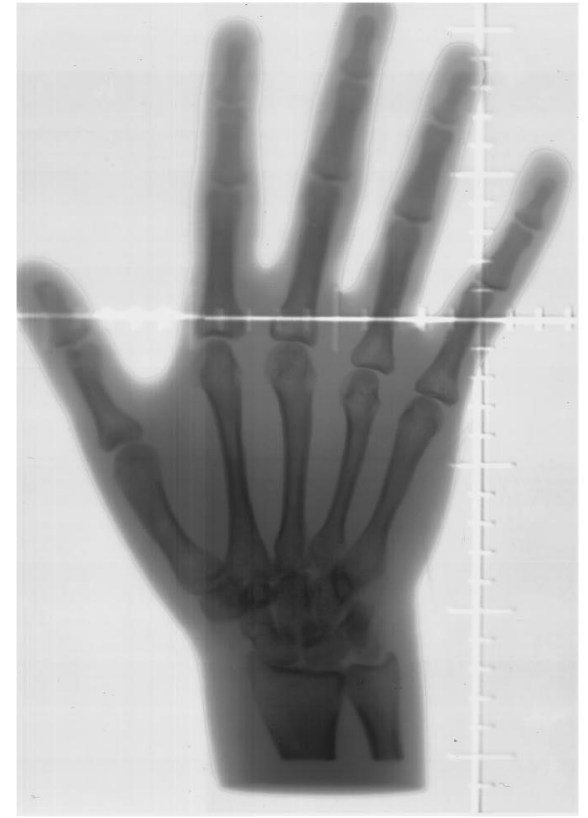

(c)

(a)

FIG. 14. X-ray images of (a) a human hand acquired in radiographic mode and of a hand phantom acquired (b) in fluoroscopic mode and (c) radiographic mode. All three images were acquired at $70 \mathrm{kVp}$ with exposures of 3.0 and $3.5 \mathrm{mAs}$ for the fluoroscopic and radiographic images, respectively.

loss of contrast is observed in the region of the group labeled $3.50 \mathrm{lp} / \mathrm{mm}$ when the image is examined on a high resolution computer monitor. This result is generally consistent with the spatial resolution expected given the choice of x-ray converter and the sampling frequency of the array. Furthermore, virtually identical results are achieved when the orientation of the pattern is rotated by $\sim 90^{\circ}$ with respect to the array and when images are acquired radiographically.

An x-ray image of a human hand acquired with a 512 $\times 1920$ pixel portion of the array is shown in Fig. 14(a). $\mathrm{X}$-ray images of an anthropomorphic hand phantom acquired in fluoroscopic and radiographic modes with the full array are shown in Figs. 14(b) and 14(c), respectively. While the upper portion of all presented images in the region of the distal phalanges suffers from blurring as a result of less than optimal contact between the screen and the array, the good spatial resolution properties of the imager are nevertheless demonstrated by the fact that the cortical margins and the joint spaces are well defined in the carpal and metacarpal regions of each image. However, by virtue of fact that trabeculation is not evident, the spatial resolution of the system is certainly less than that of a typical film-screen system. In the case of the human image, the joint spaces between the carpal bones are well defined and the entire image is as sharp as the phantom images, demonstrating that the human image could be taken in a clinically practical exposure time. The spatial and contrast information content of the fluoroscopic and radiographic phantom images appear to be virtually indistinguishable. Finally, as would be expected for this flat-panel technology, the contrast in the finger tips is the same as in the vicinity of the wrist, which is consistent with the expectation of an absence of the blooming problem that plagues image intensifier systems.

\section{DISCUSSION}

The AMFPI described in this article represents an early prototype of an emerging technology. As such, many aspects of both the physical makeup of the imager as well as its function and performance fall short of that required for practical use in a clinical setting. However, a variety of interesting implications for the future development and clinical application of this technology can be made based on the experience gained in developing this imager, from the results presented on the empirical studies of its signal performance, and from the various considerations outlined for radiographic and fluoroscopic operation.

The considerable improvement in the dark signal behavior of the pixels over that of earlier arrays is the result of extensive efforts both to characterize the origin and nature of the photodiode leakage current ${ }^{37}$ and to improve the quality of the array fabrication process. The excellent stability of the dark signal (Fig. 4) resulting from these efforts should help to ensure that, once acquired, the pixel-by-pixel gain and offset constants required for image processing remain valid for at least a day. In addition, the relatively small changes in the dark signal level with increasing frame rate [Fig. 5(a)] along with the observed independence of fluoroscopic pixel 
response with frame rate suggest that, for a given imager, only a few sets (and perhaps even a single set) of image processing constants may suffice for fluoroscopic operation across a large range of frame rates. Given the observed independence of radiographic pixel response with frame rate for a given radiographic initialization time, the same may be true for radiographic mode. A high degree of stability and wide range of applicability of image processing constants would be of significant practical benefit in a clinical setting as this would reduce the amount of time, effort, and computing resources necessary to acquire, maintain, and implement them. Moreover, the relatively minor changes in the dark signal level at extended frame times [Fig. 5(a)] suggest that imagers of this design might facilitate studies involving long exposure times at low exposure levels in applications as diverse as nuclear medicine, autoradiography, and astronomy.

The investigation of the temporal behavior of pixel sampling demonstrates that an $\sim 24 \mathrm{~cm}$ array with a $127 \mu \mathrm{m}$ pixel pitch can be designed to permit fluoroscopic operation up to $\sim 30 \mathrm{fps}$ (Fig. 7) - consistent with the results of an earlier, more general theoretical analysis. ${ }^{38}$ Even higher frame rates should be achievable through the use of TFT-on voltages larger than those used in the present study. This requires that the increased size of the switching transient (Fig. 6) be compensated for both in the design of the preamplification circuit and by adjusting $V_{\text {bias }}$ so as to maintain good efficiency of light detection (Fig. 8). However, given the modest optical fill factor of the present array and the desirability of higher fill factors (which increase the photodiode capacitance and the pixel time constant thereby reducing the maximum frame rate), maintaining video frame rates while increasing fill factor could present future design challenges.

The high degree of electrical isolation between neighboring pixels and the fact that the gate lines of the AMFPI can be read out in any arbitrary sequence allows for modes of operation in which all or part of the array is read out at full or reduced resolution. As a result, it is conceivable that a flatpanel imager based on a large-area array with an $\sim 100 \mu \mathrm{m}$ pixel pitch could be developed which would allow both highresolution radiographic imaging as well as a variety of fluoroscopic readout modes. For example, by reading out pairs of gate address lines simultaneously and combining the data from neighboring data lines digitally in the acquisition electronics, the device would deliver fluoroscopic images (e.g., at $30 \mathrm{fps}$ ) with $200 \mu \mathrm{m}$ pixel resolution-a resolution objective currently under examination by other groups developing AMFPIs. ${ }^{10,39}$ Furthermore, for the same data bandwidth (bytes per second) required to read out the entire array at half-resolution (i.e., $200 \mu \mathrm{m}$ ), any contiguous quarter of the array surface could be read out at full resolution (i.e., at 100 $\mu \mathrm{m})$ at the same frame rate. Thus, the device would deliver higher resolution fluoroscopic images of a smaller region. This would constitute a form of digital zoom and would give the imager a capability analogous to the magnification modes on x-ray image intensifier systems. Moreover, for a given data bandwidth, even higher frame rates (e.g., $60 \mathrm{fps}$ ) could be realized either through further reduction in the area of the array read (but with no reduction in pixel resolution) or through simultaneous readout of consecutive gate lines and combination of data from neighboring data lines with a loss of pixel resolution (but with no further loss of area). Investigations into the feasibility of such an array and its associated readout modes, including an examination of the potential signal-to-noise properties, ${ }^{19}$ are currently underway.

Generally, it is desirable to minimize charge trapping in radiographic mode in order to improve the ratio of signal to noise and thus image quality. While the amount of charge lost to trapping in a radiographic exposure can be quite large, careful optimization of the $a-\mathrm{Si}: \mathrm{H}$ fabrication process to minimize the density of trapping states as well as operation of the imager at a sufficiently large value of $V_{\text {bias }}$ can reduce the effect to $\sim 5 \%$ (Fig. 11). Comparison of charge release data from the current array with that from earlier arrays (Fig. 12) strongly suggests that further significant reductions in charge trapping should be possible. Furthermore, charge trapping significantly reduces the linearity of the pixel response curve for radiographic mode compared to that for fluoroscopic mode (Figs. 9 and 10). Since a linear response function involves only two image processing constants per pixel, requiring relatively straightforward dark and flood field measurements, strategies which reduce trapping and thereby extend the range of linearity decrease the overhead and complexity associated with these constants. Overall, given the relatively low levels of pixel dark signal and the near absence of dark signal drift, operating the array at higher values of $V_{\text {bias }}$ appears to be favorable for reasons of minimizing charge trapping and maximizing the light detection efficiency, pixel charge capacity, and range of linearity. Moreover, the observed dependence of charge trapping on $V_{\text {bias }}$ allows the interesting possibility of deliberate real-time enhancement or reduction of the magnitude of image lag in fluoroscopic applications through control of this operational parameter.

Despite a variety of limitations which constrain the performance of the current imaging system (e.g., high acquisition system noise, limited optical fill factor, suboptimal screen-array contact), it is encouraging that the phantom and human radiographic hand images demonstrate reasonable spatial and contrast information content under clinically relevant exposure conditions. It is also encouraging that the radiographic and fluoroscopic phantom images, acquired under similar conditions, provide similar information content. More detailed examinations of the image quality involving a variety of anatomical sites under a range of conditions are planned.

Though seemingly mundane, the mechanical and electrical design of the arrays and their acquisition systems, as well as the packaging of the entire system, remain major challenges in the development of this technology. A few of the most difficult and outstanding issues will be mentioned. The creation of high resolution arrays with larger optical fill factors is generally desirable and even necessary in some applications. ${ }^{19}$ However, for the present pixel design, increases in fill factor involve the reduction of feature sizes (e.g., the widths of lines and gaps between structures). This 
must be accomplished while keeping parasitic capacitances and pixel and line defect rates low. Thus, while considerable increases in fill factors are conceivable (to perhaps $\sim 50 \%$ at $127 \mu \mathrm{m}$ pixel pitch), fill factors closer to $100 \%$ will probably necessitate innovative array designs involving continuous sensor layers. ${ }^{13,14}$ Another issue concerns the dark noise of the present acquisition system which is relatively high compared to the levels (as low as $\sim 1000$ e rms) necessitated by various imaging applications. ${ }^{19}$ Creation of sufficiently lownoise (and low-cost) electronics, particularly the preamplifiers and the voltage supplies, is an essential, nontrivial task. In addition, present array designs require the individual connection of thousands of array address lines to external electronics in a compact, reliable, and robust manner. Ultimately, this demanding and expensive requirement may be partially circumvented by the introduction of various designs of multiplexers (made from polysilicon or some other highermobility material) directly onto the array substrate. Such onarray multiplexing would allow fewer channels of external gate driver and preamplifier electronics to control the gate address lines and read out the data lines, respectively, thereby reducing component costs. Ultimately, it may eventually prove feasible to incorporate the preamplification and even the digitization circuitry directly onto the array substrate. Finally, for all of the aforementioned reasons, the creation of imagers incorporating arrays with pixel pitches substantially less than $\sim 100 \mu \mathrm{m}$ (e.g., for mammography) is expected to be especially challenging.

\section{SUMMARY}

The development of the first large-area, high-resolution, flat-panel active matrix imager has been reported. The imager detects radiation by means of a phosphor converter and incorporates an optically sensitive, pixelated array each pixel of which comprises an a-Si:H photodiode and thin-film transistor. Considerations for the operation of the imager have been discussed in the context of the operational parameters (consisting of voltages and timings) which control the imager. The first comprehensive examination, as a function of these operational parameters, of the pixel signal properties of such an imager operated in fluoroscopic and radiographic modes as well as in the dark has been reported. A variety of fluoroscopic and radiographic images, including a radiograph of a human hand, have been presented. The results of these initial investigations are encouraging and support the hypothesis that this technology could eventually offer a clinically useful tool for fluoroscopic and radiographic x-ray imaging applications.

\section{ACKNOWLEDGMENTS}

We wish to thank R. Weisfield, Ph.D., Xerox, PARC for providing the absolute photodiode efficiencies and $\mathrm{D}$. Trauernicht, Ph.D., Kodak Health Imaging Systems, for providing a variety of phosphor screens and for insightful discussions concerning the measurements. We also thank H.-P. Chan, Ph.D., M. M. Goodsitt, Ph.D., and M. V. Marx, M.D. for comments on the manuscript and K.-W. Jee and H. Schek
III for assistance with the figures. This work is supported by the National Institutes of Health Grant No. R01-CA56135.

${ }^{1}$ R. A. Street, S. Nelson, L. E. Antonuk, and V. Perez Mendez, "Amorphous silicon sensor arrays for radiation imaging," Mater. Res. Soc. Symp. Proc. 192, 441-452 (1990).

${ }^{2}$ L. E. Antonuk, J. Boudry, W. Huang, D. L. McShan, E. J. Morton, J. Yorkston, M. J. Longo, and R. A. Street, “Demonstration of megavoltage and diagnostic x-ray imaging with hydrogenated amorphous silicon arrays,' Med. Phys. 19, 1445-1466 (1992).

${ }^{3}$ K. Kobayashi, S. Makida, Y. Sato, and T. Hamano, " $640 \times 400$ pixel a-Si:H TFT driven 2-dimensional image sensor,' Proc. SPIE 1900, 40-46 (1993).

${ }^{4}$ M. J. Powell, I. D. French, J. R. Hughes, N. C. Bird, O. S. Davies, C. Glasse, and J. E. Curran, "Amorphous silicon image sensor arrays," Mater. Res. Soc. Symp. Proc. 258, 1127-1137 (1992).

${ }^{5}$ M. Yamaguchi, Y. Kaneko, and K. Tsutsui, "Two-dimensional contacttype image sensor using amorphous silicon photo-transistor,', Jpn. J. Appl. Phys. 32, 458-461 (1993).

${ }^{6}$ D. L. Lee, L. K. Cheung, and L. S. Jeromin, “A new digital detector for projection radiography,', Proc. SPIE 2432, 237-249 (1995).

${ }^{7}$ W. Zhao and J. A. Rowlands, "X-ray imaging using amorphous selenium: Feasibility of a flat panel self-scanned detector digital radiology,', Med. Phys. 22, 1595-1604 (1995).

${ }^{8}$ C. van Berkel, N. C. Bird, C. J. Curling, and I. D. French, "'2D image sensor arrays with nip diodes,' Mater. Res. Soc. Symp. Proc. 297, 939944 (1993).

${ }^{9}$ K. Yamamoto, K. Sai, Y. Ohta, H. Mimura, and K. Kitamura, “Amorphous silicon 2-dimensional image sensor," Technical Digest of the 8th Sensor Symposium, 1989 (unpublished), pp. 55-58.

${ }^{10}$ J. Chabbal, C. Chaussat, T. Ducourant, L. Fritsch, J. Michailos, V. Spinnler, G. Vieux, M. Arques, G. Hahm, M. Hoheisel, H. Horbaschek, R. Schulz, and M. Spahn, "Amorphous silicon x ray image sensor,' Proc. SPIE 2708, 499-510 (1996).

${ }^{11}$ T. Graeve, W. Huang, S. M. Alexander, and Y. Li, “Amorphous silicon image sensor for x-ray applications,'” Proc. SPIE 2415, 177-181 (1995).

${ }^{12} \mathrm{~W}$. Zhao and J. A. Rowlands, "A large area solid-state detector for radiology using amorphous selenium,'’ Proc. SPIE 1651, 134-143 (1992).

${ }^{13}$ L. E. Antonuk, J. Boudry, J. Yorkston, E. J. Morton, W. Huang, and R. A. Street, "Development of thin-film, flat-panel arrays for diagnostic and radiotherapy imaging, ',Proc. SPIE 1651, 94-105 (1992).

${ }^{14}$ R. A. Street, X. D. Wu, R. Weisfield, S. Ready, R. Apte, M. Nguyen, and P. Nylen, "Two dimensional amorphous silicon image sensor arrays," Mater. Res. Soc. Symp. Proc. 377, 757-765 (1995).

${ }^{15}$ L. E. Antonuk, J. Boudry, Y. El-Mohri, W. Huang, J. Siewerdsen, and J. Yorkston, "A high resolution, high frame rate, flat-panel TFT array for digital x-ray imaging,' Proc. SPIE 2163, 118-128 (1994).

${ }^{16}$ L. E. Antonuk, "Flat (-panel) horizons in digital x-ray imaging,' Photonics Spectra, June 1995 (unpublished), pp. 108-114.

${ }^{17}$ L. E. Antonuk, J. M. Boudry, Y. El-Mohri, W. Huang, J. H. Siewerdsen, J. Yorkston, and R. A. Street "Large area, flat-panel amorphous silicon imagers,'’ Proc. SPIE 2432, 216-227 (1995).

${ }^{18}$ L. E. Antonuk, Y. El-Mohri, W. Huang, J. Siewerdsen, J. Yorkston, and R. A. Street, "A large area, high-resolution $a$-Si:H array for X-ray imaging,’ Mater. Res. Soc. Symp. Proc. 336, 855-860 (1994).

${ }^{19}$ J. H. Siewerdsen, L. E. Antonuk, Y. El-Mohri, J. Yorkston, W. Huang, J. M. Boudry, and I. A. Cunningham, "Empirical and theoretical investigation of the noise performance of indirect detection, active matrix flatpanel imagers (AMFPIs) for diagnostic radiology,', Med. Phys. 24, 71-89 (1997).

${ }^{20}$ J. Yorkston, L. E. Antonuk, N. Seraji, W. Huang, J. H. Siewerdsen, and Y. El-Mohri, "MTF measurements with high resolution $a-\mathrm{Si}: \mathrm{H}$ imaging arrays," Proc. SPIE 2432, 260-269 (1995).

${ }^{21}$ L. E. Antonuk, J. Boudry, C. W. Kim, M. J. Longo, E. J. Morton, J. Yorkston, and R. A. Street, "Signal, noise and readout considerations in the development of amorphous silicon photodiode arrays for radiotherapy and diagnostic x-ray imaging,' Proc. SPIE 1443, 108-119 (1991).

${ }^{22}$ R. A. Street, “Amorphous silicon electronics,' Mater. Res. Soc. Bull. XVII, 70-76 (1992).

${ }^{23}$ L. E. Antonuk, J. Yorkston, W. Huang, J. Boudry, E. J. Morton, and R. A. Street, "Large area, flat-panel $a-\mathrm{Si}: \mathrm{H}$ arrays for x-ray imaging," Proc. SPIE 1896, 18-29 (1993). 
${ }^{24}$ L. E. Antonuk, J. Yorkston, W. Huang, H. Sandler, J. Siewerdsen, and Y. El-Mohri, "Megavoltage imaging with a large area, flat-panel, amorphous silicon imaging array," Int. J. Radiat. Onc. Biol. Phys. 36, 661672 (1996).

${ }^{25}$ E. J. Morton, L. E. Antonuk, J. E. Berry, W. Huang, P. Mody, and J. Yorkston, "A data acquisition system for flat-panel imaging arrays," IEEE Trans. Nucl. Sci. 41, 1150-1154 (1994).

${ }^{26} \mathrm{R}$. A. Street, "Long time transient conduction in $a$-Si:H p-i-n devices," Philos. Mag. 63, 1343-1363 (1991).

${ }^{27}$ J. Yorkston, L. E. Antonuk, E. J. Morton, J. Boudry, W. Huang, C. W. Kim, M. J. Longo, and R. A. Street, "The dynamic response of hydrogenated amorphous silicon imaging pixels," Mater. Res. Soc. Symp. Proc. 219, 173-178 (1991).

${ }^{28}$ R. A. Street, Hydrogenated Amorphous Silicon (Cambridge University Press, Cambridge, 1991).

${ }^{29}$ R. A. Street, R. L. Weisfield, R. B. Apte, S. E. Ready, A. Moore, M. Nguyen, W. B. Jackson, and P. Nylen, "Amorphous silicon sensor arrays for X-ray and document imaging," (unpublished).

${ }^{30}$ R. A. Street, I. Fujieda, R. Weisfield, S. Nelson, and P. Nylen, "Large area 2-dimensional $a$-Si:H imaging arrays," Mater. Res. Soc. Symp. Proc. 258, 1145-1150 (1992).

${ }^{31}$ Y. Matsunaga, F. Hatori, T. Hiroyuki, and O. Yoshida, "Analysis of signal to noise ratio of photoconductive layered solid-state imaging device," IEEE Trans. Electron Devices 42, 38-42 (1995).

${ }^{32}$ J. Yorkston, L. E. Antonuk, W. Huang, and R. A. Street, "Photoresponse linearity of $a$-Si:H imaging pixels," Mater. Res. Soc. Symp. Proc. 297, 951-956 (1993)

${ }^{33}$ L. E. Antonuk, J. Siewerdsen, J. Yorkston, and W. Huang, "Radiation response of amorphous silicon imaging arrays at diagnostic energies," IEEE Trans. Nucl. Sci. 41, 1500-1505 (1994).
${ }^{34}$ J. Yorkston, L. E. Antonuk, N. Seraji, J. Boudry, W. Huang, E. J. Morton, and R. A. Street, "Comparison of computer simulations with measurements from $a$-Si:H imaging arrays," Mater. Res. Soc. Symp. Proc. 258, 1163-1168 (1992).

${ }^{35}$ L. E. Antonuk, J. Yorkston, W. Huang, J. H. Siewerdsen, J. M. Boudry, Y. El-Mohri, and M. V. Marx, "A real-time, flat-panel, amorphous silicon, digital x-ray imager," Radiographics 15, 993-1000 (1995).

${ }^{36}$ L. E. Antonuk, J. Yorkston, W. Huang, J. Boudry, E. J. Morton, M. Longo, and R. A. Street, "Factors affecting image quality for megavoltage and diagnostic x-ray $a$-Si:H imaging arrays," Mater. Res. Soc. Symp. Proc. 258, 1069-1074 (1992).

${ }^{37}$ E. A. Schiff, R. A. Street, and R. L. Weisfield, "Reverse bias currents in amorphous silicon nip sensors," J. Non-Cryst. Solids 198-200, 11551158 (1996).

${ }^{38}$ L. E. Antonuk, J. Yorkston, W. Huang, J. Siewerdsen, and R. A. Street, "Considerations for high frame rate operation of two-dimensional $a$-Si:H imaging arrays,"' Mater. Res. Soc. Symp. Proc. 297, 945-950 (1993).

${ }^{39}$ U. W. Schiebel, N. Conrads, N. Jung, M. Weibrecht, H. Wieczorek, T. T. Zaengel, M. J. Powell, I. D. French, and C. Glasse, "Fluoroscopic x-ray imaging with amorphous silicon thin-film arrays," Proc. SPIE 2163, 129-140 (1994).

${ }^{40}$ J. M. Boudry and L. E. Antonuk, "Radiation damage of amorphous silicon, thin-film, field-effect transistors," Med. Phys. 23, 743-754 (1996).

${ }^{41}$ T. S. Curry, J. E. Dowdey, and R. C. Murry, Christensen's Physics of Diagnostic Radiology, 4th ed. (Lea \& Febiger, 1990), p. 162.

${ }^{42}$ H. Wieczorek, G. Frings, P. Quadflieg, U. Schiebel, T. F. Bergen, F. M. Dreesen, M. A. C. Ligtenberg, and T. Poorter, "CsI:Tl for solid state x-ray detectors," International Conference on Inorganic Scintillators and their Applications (Delft, Netherlands, 1995) (unpublished). 